\title{
Sm-Nd and Rb-Sr Datings, Petrogenesis and Thermometry of the Ngovayang Area (South-West Cameroon): Isotopic Data Insight of Recycling Crust and Convergence Orogen
}

\author{
Ndong Bidzang Francois ${ }^{1, ~ *, ~ N t o m b a ~ S y l v e s t r e ~}$ Martial $^{2}$, Ntomb Yvan Demonstel ${ }^{2}$, \\ Messi Ottou Eric Jose ${ }^{3}$, Magnekou Takamte Rufine Christelle ${ }^{3}$ \\ ${ }^{1}$ Ore Processing Laboratory, Institute for Geological and Mining Research, Yaounde, Cameroon \\ ${ }^{2}$ Centre for Geological and Mining Research, Institute for Geological and Mining Research, Yaounde, Cameroon \\ ${ }^{3}$ Department of Earth Sciences, University of Yaounde I, Yaounde, Cameroon
}

Email address:

fndongbidzang@gmail.com (N. B. Francois)

${ }^{*}$ Corresponding author

\section{To cite this article:}

Ndong Bidzang Francois, Ntomba Sylvestre Martial, Ntomb Yvan Demonstel, Messi Ottou Eric Jose, Magnekou Takamte Rufine Christelle. Sm-Nd and Rb-Sr Datings, Petrogenesis and Thermometry of the Ngovayang Area (South-West Cameroon): Isotopic Data Insight of Recycling Crust and Convergence Orogen. Science Research. Vol. 7, No. 2, 2019, pp. 17-32. doi: 10.11648/j.sr.20190702.12

Received: August 5, 2019; Accepted: September 4, 2019; Published: September 19, 2019

\begin{abstract}
The Ngovayang trondhjemitic gneisses belong to the Nyong Complex at the Northwest boundary of the Ntem Complex. A combined study of whole-rock major, trace elements and isotopic data, $\mathrm{Sm}-\mathrm{Nd}$ and $\mathrm{Rb}-\mathrm{Sr}$ indicate that trondhjemitic gneisses are peraluminous and range from weakly I-type to S-type with nearly constant A/CNK values between 1.04 and 1.24. Their I- and S- type characters suggest that juvenile materials have been partially remobilized or recycled. These trondhjemitic gneisses have slightly to moderate $\mathrm{MgO}, \mathrm{Cr}$ and Ni contents, $\mathrm{ISr}(0.703677$ - 0.741911) and low $\varepsilon \mathrm{Nd}(\mathrm{t})($ from 16.48 to -10.6) values. Such geochemical features suggest a small mantle-source contribution, coupled with assimilation of some upper and lower crustal materials and indicate the implication of old crust probably with both Archean and Early eburnean origin $(2597-2318 \mathrm{Ma})$. They also exhibit geochemical features typical of calc-alkaline, crustal contaminated calcalkaline rocks, volcanic arc and trondhjemitic nature. These results show that old crust has recycled and trondhjemitic gneisses are linked to convergent geodynamic system. The petrogenetic diagrams of $\mathrm{AFM}$ vs $\mathrm{CFM}, \mathrm{MgO}$ vs $\mathrm{SiO}_{2}$ and $\mathrm{Rb} / \mathrm{Ba}$ vs $\mathrm{Rb} / \mathrm{Sr}$ and lower $\mathrm{K}_{2} \mathrm{O} / \mathrm{Na}_{2} \mathrm{O}$ ratio values $(0.38-0.51)$, indicate that the magmatic source materials are mainly composed of plagioclase-rich sources such as metagreywackes - metabasic mixtures, metagreywackes and metabasics and basalt or igneous rocks. These sources materials occurring in the melt originated from both slab melting and assimilation of upper and lower crust at ca. $732.4-928.7^{\circ} \mathrm{C}$ and at $2597-2318 \mathrm{Ma}$. This study shows that Archean crust may have a North-West extension beyond the actual boundary with Nyong Complex.
\end{abstract}

Keywords: Nyong Complex, Ngovayang Trondhjemitic Gneisses, Sm-Nd and Rb-Sr Datings, Thermometry, Convergent Geodynamic System

\section{Introduction}

The Nyong Complex is one of eburnean continental portion in the world [1-2], which is made up of tonalite, trondhjemite and granodiorite and other granitoids associated to basic rocks [1-5]. In the field, trondhjemites commonly display a gneissic appearance (grey gneisses), often migmatitic and can be divided into three groups: Amp-Grt bearing orthogneiss, Bt-Opx bearing orthogneiss and Opx bearing orthogneiss. These rocks can provide information on tectonic, thermal evolution and crustal genesis and growth of orogenic belts [6-7]. Certain parameters like aluminum saturation index and geochemical variability obtained by these trondhjemite gneisses permit to know their source composition, information on magmatic differentiation [8] and temperature of crystallization and growth [9]. Many varieties of geodynamic settings have been proposed such as 
subduction, continental collision [6, 10-13] and extensional environments. The West Central African Belt (WCAB) is located along the western side of the Congo craton from Angola to Cameroon [14], and continues to Brazil as transamazonian belt [15]. The present work focuses on the Ngovayang massif which belong to Nyong serie actually called Nyong Complex [16-17], and is situated at the northwest beyond the Ntem Complex. During Eburnean cycle, convergence and collision of two segment Cratons probably resulted in the formation of an important mountain belt which is Ngovayang (Figure 1c). This mountain constitues part of the study area (Figure 1d). Several studies on Paleoproterozoic rocks from the West Central African Belt (WCAB) have been published [1, 4, 14, 16-19], but none of them have paid attention of rocks exposed atop of Ngovayang massif probably because of its inaccessibility (with high relief, $>1000 \mathrm{~m}$, Figure 1c) coupled with lateritic layer and important vegetation covering the outcrop. Thus studies are concentrated elsewhere or on the massif surroundings. The Cameroon Eburnean belts are represented by Nyong and Ayna series [20-21], which correspond to area of dominant crustal recycling of the neighbouring Ntem Complex [3-4] and area of crustal growth within Neoproterozoic belt in the North of Ntem Complex [19, 2224]. Other studies show that, this continental crust result from the convergence and collision between the Sao Francisco-Nigeria shield bloc and a former Congo megacraton [1, 19]. In spite of studies in the Nyong Complex, the boundary of these two complexes remains matter of debate. The estimations of temperature have been done by qualitative methods with mineral presents in thin sections. Thus we have high grade granulitic assemblages [19] and amphibolitic climate [16] that peaked at ca. 2050 Ma $[4,14,20]$, in the Nyong Complex.
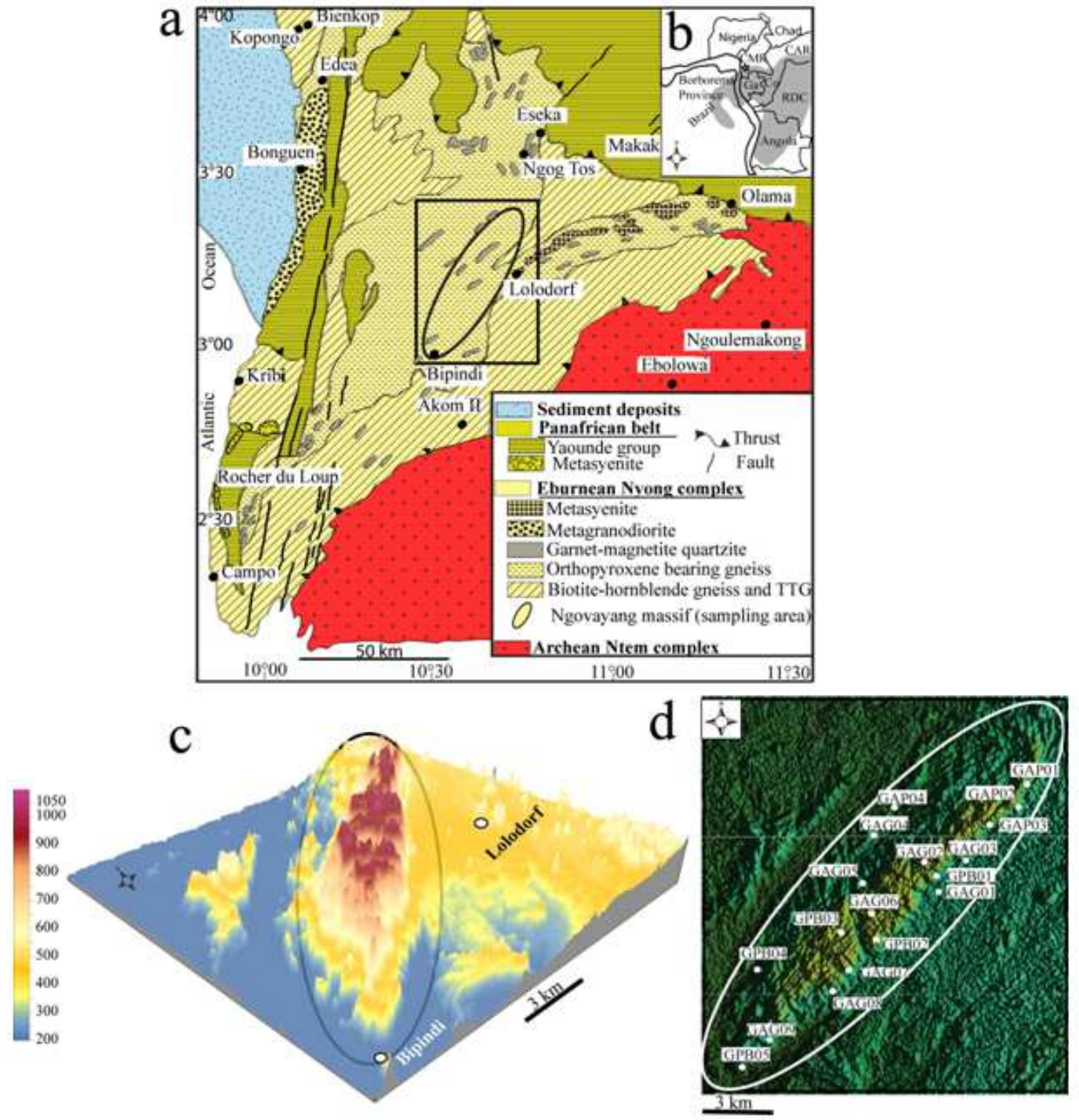

Figure 1. a-Simplified geological map of the Nyong Complex Southwestern Cameroon (modified after [1, 63], showing study area (ellipsoid) and his neighborhoods (rectangle); b-Gondwana map portion showing limit of Congo and Sao Francisco assemblage (grey area) between Central Africa and NE Brazil (after [1, 63] and Nyong Complex; c-Topographical map of Ngovayang massif (study area, ellipsoid) and his neighborhoods and d-Ngovayang massif map (ellipsoid) with sample locations. 
In this paper, we present for the first time whole rock geochemical data, isotopic data, $\mathrm{Sm}-\mathrm{Nd}$ datings of Ngovayang orthogneisses and aim to determine the source composition or petrogenesis, melting and evolving temperatures. We have a good opportunity to discuss about whether the Ngovayang area has been reworked during the Paleoproterozoic or if he represents the plate above subduction area it represents.

\section{Geological Setting}

The Eburnean orogeny in Cameroon includes Nyong complex [16-17] along the NW boundary with Ntem Complex [16-17, 25], Ayna serie [20-21], and Paleoproterozoic remnant cropping out to Bafia group and Adamawa-yade domain which are the late Neoproterozoic Panafrican fold belt [22-24]. Formations on Nyong gneisses $[4,26]$ include biotite-hornblende gneiss sometimes locally defined as grey gneiss (or migmatic grey gneiss) of TTG composition [3], orthopyroxene-garnet gneiss interpreted as charnockitic composition, garnet-amphibole pyroxenite, banded iron formation and magmatic rocks (syn to late charnockite, augen metadiorite, syenite, granite, granulite, eclogite and dolerite; [1-2, 19]. Our study focuses on orthogneisses which include orthopyroxene gneiss (Figure 1a, [1] or many variable types of gneiss differentiated by mineralogy proportions and composed of Amp-Grt bearing gneiss, Opx bearing gneiss and $\mathrm{Bt}$ - Opx bearing gneiss. Generally, the Nyong Complex is characterized by flat lying foliation associated with a variably oriented stretching lineation which results from the transposition of primary foliation by isoclinal recumbent folds appearing in phase two during deformation. This foliation is disturbed locally by large opened folds associated with shear zone [1, 19]. The two tectonic phases which are described in this Complex are synchronous with the charnockite formation (near the boundary with Ntem Complex) and/or migmatization. This migmatite occurs parallel to regional foliation and along the late shearzone during phase two. The metamorphic evolution is polycyclic with high grade granulitic assemblages marked by fine grained of polygonal quartzofeldspathic minerals which suggest high temperature recrystallization and the presence of corona rims which illustrates a static evolution under granulite to amphibolite facies conditions. This retrogressive phase continues until it reaches greenschist facies conditions which are locally overprinted [3, 27]. This phase two is known as belonging to the Eburnean nappe formations which were transported top to east onto the Ntem Complex under the amphibolitic climate [16] and peaked at ca. $2050 \mathrm{Ma}[4,14,27]$. Based on geochemical studies, the migmatitic gneisses (belonging to TTG suite) are comparable with those of Ntem Complex [3]. The critical isotopic data indicate that: the Nyong rocks were formed from both contribution of Archean protolith and juvenile material as demonstrated by Sm-Nd and $\mathrm{T}_{\mathrm{DM}}$ ages $[4,18]$. These Nyong rocks have also experienced a major late tectonic, granulitic and migmatitic events at ca. 2.05 Ga. The Nyong Complex may be interpreted as a proximal area characterized by reworking and recycling of adjacent Archean cratonic crust [19].

\section{Samples and Analytical Techniques}

Eighteen samples of Ngovayang Eburnean trondhjemitic gneiss rocks have been studied. These samples are localized in the Ngovayang massif (Figure 1d) and belong to three main gneiss types: Amp-Grt gneiss (GAG01, GA02, GA03, GA04, GA05, GA06, GA07, GA08 and GA09); Bt - Opx gneiss (GPB01, GPB02, GPB03 and GPB04) and Opx gneiss (GAP01, GAP02, GAP03 and GAP04) (table 1). Whole rock compositions from the representative samples of Ngovayang trondhjemitic gneiss were determined using Phillips PW 1840 X-ray fluorescence (XRF) for major elements and Induced Couple Plasma Mass Spectrometry (ICP-MS) on a VG-plasma Quad STE ICP mass spectrometer for trace elements in the OMAC laboratories of the ALS Geochemistry group in Ireland. Isotope geochemical analyses were achieved place at the University of Rennes in France. The analyses were carried out following the isotopic dilution method. Based on the method, the determination of isotopic composition of samples was done using a multicollector mass spectrometer by thermos-ionization of the Finnigam MAT 262 type. The reactants used to dissolve the samples were distilled twice in the case of Rb-Sr and Sm$\mathrm{Nd}$ systems. The dissolution of samples (100 to $200 \mathrm{mg}$ on average) was done in a Teflon spray (Sanillex) with a mixture of concentrated $\mathrm{HNO}_{3}(7 \mathrm{~N})+\mathrm{HF}$ on a hot plate at $150^{\circ} \mathrm{C}$ for 24 to 48 hours. That process was repeated a second time after evaporation. After this second process, the sample was evaporated and then dissolved in $6 \mathrm{~N}$ of acid chlorite. For the identification of elements ( $\mathrm{Rb}, \mathrm{Sr}, \mathrm{Nd}) \mathrm{a}$ tracer (or Spike) was used. Nd and Sm isotope used double tracer ${ }^{149} \mathrm{Sm}-{ }^{150} \mathrm{Nd}$, Sr used one tracer ${ }^{84} \mathrm{Sr}$ and one for $\mathrm{Rb}$, ${ }^{87} \mathrm{Rb}$. Relative to $\mathrm{Rb}-\mathrm{Sr}$, the dissolved sample was loaded on a column of $14 \mathrm{ml}$ volume containing cationic resin 50AGX8 200-400 mesh. Rb was obtained after an elution $35 \mathrm{ml}$ and $\mathrm{Sr}$ after $75 \mathrm{ml}$ of $2 \mathrm{NHCl}$. Relative to $\mathrm{Rb}-\mathrm{Sr}$, sample dissolute was loaded on a column of $14 \mathrm{ml} \mathrm{b}$ volume of a cationic resin 50AGX8 200-400 mesh. The $\mathrm{Rb}$ was obtained after an elution of $35 \mathrm{ml}$ and $\mathrm{Sr}$ after $75 \mathrm{ml}$ of $2 \mathrm{NHCl}$. For $\mathrm{Sm}$ and $\mathrm{Nd}$, the rare earth elements (light) were obtained after the collection of Sr with $40 \mathrm{ml}$ HCL6N. The selective separation of $\mathrm{Nd}$ and $\mathrm{Sm}$ required a second column of $3 \mathrm{ml}$ by volume of Teflon powder treated with phosphoric acid, 2(diethylhexil). The samples were deposited in a $0.25 \mathrm{ml}$ HCL acid column of $0.15 \mathrm{~N}$. The $\mathrm{Nd}$ was recovered after about $20 \mathrm{ml}$ of $0.15 \mathrm{NHCL}$ while Sm recovery was after 15 $\mathrm{ml}$ of about $0.3 \mathrm{NHCL}$. The $\mathrm{Sr}$ and $\mathrm{Nd}$ isotopic ratios were corrected for mass fractionation by normalizing to ${ }^{86} \mathrm{Sr} /{ }^{88} \mathrm{Sr}=$ 0.1195 and ${ }^{146} \mathrm{Nd} /{ }^{144} \mathrm{Nd}=0.7219$, respectively. Total procedural blanks were b160 pg for $\mathrm{Sr}$ and b80 pg for $\mathrm{Nd}$. Replicate analyses of the NBS-987 Sr standard during the 
course of this study yielded a mean value of ${ }^{87} \mathrm{Sr} /{ }^{86} \mathrm{Sr}$ $=0.710259 \pm 10(2 \sigma)$. Measurements of the Ames $\mathrm{Nd}$ standard gave a mean ${ }^{143} \mathrm{Nd} /{ }^{144} \mathrm{Nd}$ ratio of $0.512125 \pm 8(2 \sigma$, $\mathrm{n}=5) .{ }^{87} \mathrm{Rb} /{ }^{86} \mathrm{Sr}$ ratios of whole-rock samples were calculated based on the measured ${ }^{87} \mathrm{Sr} /{ }^{86} \mathrm{Sr}$ ratios and the $\mathrm{Rb}$ and $\mathrm{Sr}$ concentrations determined by XRF. Errors are given in 2 sigma (95\%) confidence level. using the ISOPLOT/Excel program [28]. The CIPW normative compositions, temperature liquidus and magma density were obtained from the program written by Kurt Hollocher and by calculation method from [29], (table 2). Temperatures were obtained by GCDKit [30] and excel spreadsheet programs using major elements $\left(\mathrm{SiO}_{2}, \mathrm{P}_{2} \mathrm{O}_{5}, \mathrm{TiO}_{2}, \mathrm{Al}_{2} \mathrm{O}_{3}\right.$, Zirconium concentration) and LREE ( $\mathrm{La}, \mathrm{Ce}$ ) in the [31] apatite equation; [32] zircon equation, [33] rutile equation and measured $\mathrm{Al}_{2} \mathrm{O}_{3} / \mathrm{TiO}_{2}$ ratio [34]. All these equations are reported in GCDKit manual [30].

Table 1. Chemical data of the Ngovayang throndhjemitic gneisses (NTG).

\begin{tabular}{|c|c|c|c|c|c|c|c|c|c|}
\hline \multirow{2}{*}{ Samples } & \multicolumn{9}{|c|}{ Amp-Grt bearing Orthogneiss } \\
\hline & GAG01 & GAG02 & GAG03 & GAG04 & GAG05 & GAG06 & GAG07 & GAG08 & GAG09 \\
\hline $\mathrm{SiO}_{2}$ & 69.87 & 70.5 & 71.41 & 71.4 & 70.74 & 70.18 & 71.42 & 70.4 & 71.76 \\
\hline $\mathrm{TiO}_{2}$ & 0.29 & 0.29 & 0.19 & 0.56 & 0.35 & 0.28 & 0.39 & 0.41 & 0.13 \\
\hline $\mathrm{Al}_{2} \mathrm{O}_{3}$ & 14.75 & 14.23 & 14.07 & 14.25 & 14.53 & 14.22 & 14.62 & 15.04 & 14.6 \\
\hline $\mathrm{Fe}_{2} \mathrm{O}_{3}$ & 2.62 & 2.66 & 1.94 & 1.94 & 2.85 & 2.52 & 2.38 & 2.32 & 2.46 \\
\hline $\mathrm{MnO}$ & 0.08 & 0.08 & 0.05 & 0.09 & 0.04 & 0.02 & 0.05 & 0.01 & 0.02 \\
\hline $\mathrm{MgO}$ & 1.34 & 1.34 & 0.98 & 1.01 & 1.43 & 1.28 & 1.18 & 1.13 & 1.21 \\
\hline $\mathrm{Na}_{2} \mathrm{O}$ & 5.8 & 6.47 & 5.28 & 5.41 & 5.49 & 5.64 & 5.07 & 4.94 & 5.05 \\
\hline $\mathrm{K}_{2} \mathrm{O}$ & 2.25 & 3.1 & 2.49 & 2.75 & 2.06 & 2.76 & 2.38 & 2.77 & 2.36 \\
\hline $\mathrm{P}_{2} \mathrm{O}_{5}$ & 0.19 & 0.05 & 0.17 & 0.21 & 0.11 & 0.1 & 0.15 & 0.07 & 0.07 \\
\hline LOI & 0.7 & 0.66 & 0.71 & 0.75 & 0.73 & 0.97 & 0.64 & 0.65 & 0.66 \\
\hline TOTAL & 100.1 & 101.02 & 99.02 & 99.79 & 100.14 & 99.88 & 100.35 & 100.02 & 100.38 \\
\hline $\mathrm{K}_{2} \mathrm{O} / \mathrm{Na}_{2} \mathrm{O}$ & 0.39 & 0.48 & 0.47 & 0.51 & 0.38 & 0.49 & 0.47 & 0.56 & 0.47 \\
\hline $\mathrm{CaO} / \mathrm{Na}_{2} \mathrm{O}$ & 0.38 & 0.25 & 0.33 & 0.26 & 0.33 & 0.34 & 0.41 & 0.46 & 0.41 \\
\hline $\mathrm{A} / \mathrm{NK}$ & 1.23 & 1.02 & 1.29 & 1.2 & 1.29 & 1.16 & 1.34 & 1.35 & 1.34 \\
\hline $\mathrm{Ba}$ & 571 & 757 & 552 & 302 & 516 & 425 & 676 & 557 & 1040 \\
\hline $\mathrm{Rb}$ & 54 & 29 & 48 & 49 & 40 & 51 & 44 & 42 & 51 \\
\hline $\mathrm{Sr}$ & 400 & 870 & 690 & 308 & 317 & 399 & 655 & 620 & 590 \\
\hline $\mathrm{Zr}$ & 34 & 106 & 165 & 78 & 83 & 76 & 87 & 49 & 129 \\
\hline $\mathrm{Nb}$ & 0.1 & 2.5 & 3.2 & 6.8 & 5.6 & 4.34 & 7.21 & 3.99 & 0.98 \\
\hline $\mathrm{Ni}$ & 5 & 31 & 19 & 20 & 18 & 21 & 17 & 33 & 36 \\
\hline $\mathrm{Co}$ & 2.3 & 1.2 & 1.1 & 6 & 7 & 6 & 5.4 & 1.3 & 2 \\
\hline $\mathrm{Zn}$ & 26 & 23 & 32 & 33 & 15 & 35 & 28 & 8 & 49 \\
\hline $\mathrm{Cr}$ & 63 & 77 & 94 & 10 & 43 & 65 & 72 & 53 & 148 \\
\hline $\mathrm{Y}$ & 8 & 4 & 7 & 6 & 5 & 7 & 10 & 12 & 10 \\
\hline Cs & 0.67 & 0.53 & 0.76 & 0.63 & 0.51 & 0.59 & 0.48 & 0.58 & 0.63 \\
\hline $\mathrm{Ta}$ & 0.67 & 6.23 & 0.43 & 0.72 & 7.21 & 0.58 & 0.63 & 6.23 & 4.05 \\
\hline Hf & 4.01 & 3.98 & 4.34 & 4.02 & 3.13 & 4.13 & 4.01 & 3.08 & 3.52 \\
\hline $\mathrm{Th}$ & 5 & 8 & 1 & 2.5 & 2.2 & 3 & 2.3 & 2 & 6 \\
\hline $\mathrm{Ga}$ & 0.07 & 2.26 & 3.02 & 4.35 & 6.75 & 4.32 & 8.86 & 1.41 & 5.43 \\
\hline $\mathrm{La}$ & 10.3 & 10.3 & 11 & 10.6 & 10.8 & 11.1 & 9.7 & 10.3 & 11.2 \\
\hline $\mathrm{Ce}$ & 25.3 & 30.29 & 27.32 & 23.32 & 27.4 & 29.32 & 24.3 & 23.9 & 25.3 \\
\hline $\operatorname{Pr}$ & 43 & 47 & 21 & 57 & 61 & 56 & 27 & 62 & 37 \\
\hline $\mathrm{Nd}$ & 11.6 & 12.2 & 10.8 & 10.4 & 11.2 & 10.9 & 10.1 & 9.3 & 10.4 \\
\hline $\mathrm{Sm}$ & 2.2 & 2.6 & 1.8 & 1 & 1.6 & 1 & 1.8 & 1.2 & 1.4 \\
\hline $\mathrm{Eu}$ & 0.6 & 0.7 & 0.6 & 0.7 & 0.4 & 0.7 & 0.6 & 0.3 & 0.6 \\
\hline $\mathrm{Gd}$ & 1.4 & 0.7 & 2 & 2 & 1.4 & 1.7 & 0.8 & 1.4 & 1.8 \\
\hline $\mathrm{Tb}$ & 0.1 & 0.3 & 0.1 & 0.3 & 0.3 & 0.1 & 0.1 & 0.2 & 0.3 \\
\hline Dy & 1.2 & 0.5 & 1.5 & 0.6 & 1.5 & 1.5 & 0.8 & 1.1 & 1.3 \\
\hline Ho & 0.1 & 0.3 & 0.2 & 0.2 & 0.1 & 0.3 & 0.1 & 0.1 & 0.2 \\
\hline $\mathrm{Er}$ & 0.6 & 0.5 & 0.4 & 0.2 & 0.1 & 0.5 & 0.5 & 0.7 & 0.3 \\
\hline $\mathrm{Tm}$ & 0.1 & 0.1 & 0.1 & 0.1 & 0.1 & 0.1 & 0.1 & 0.1 & 0.1 \\
\hline $\mathrm{Yb}$ & 0.6 & 0.5 & 0.4 & 0.7 & 0.4 & 0.5 & 0.5 & 0.7 & 0.4 \\
\hline $\mathrm{Lu}$ & 0.1 & 0.1 & 0.2 & 0.1 & 0.1 & 0.1 & 0.1 & 0.1 & 0.1 \\
\hline $\mathrm{Eu} / \mathrm{Eu}^{*}$ & 1.044 & 1.584 & 0.966 & 1.511 & 1.02 & 1.639 & 1.527 & 1.18 & 1.21 \\
\hline$(\mathrm{La} / \mathrm{Yb}) \mathrm{N}$ & 11.59 & 13.9 & 18.56 & 10.22 & 18.22 & 14.98 & 13.09 & 9.93 & 18.9 \\
\hline
\end{tabular}


Table 1. Continued.

\begin{tabular}{|c|c|c|c|c|c|c|c|c|c|}
\hline \multirow{2}{*}{ Samples } & \multicolumn{5}{|c|}{ Opx-Bt bearing Orthogneiss } & \multicolumn{4}{|c|}{ Opx bearing Orthogneiss } \\
\hline & GPB01 & GPB02 & GPB03 & GPB04 & GPB05 & GAP01 & GAP02 & GAP03 & GAP04 \\
\hline $\mathrm{SiO}_{2}$ & 70.2 & 70.09 & 70.44 & 68.08 & 70.7 & 70.31 & 70.79 & 69.86 & 69.65 \\
\hline $\mathrm{TiO}_{2}$ & 0.23 & 0.17 & 0.34 & 0.49 & 0.25 & 0.31 & 0.18 & 0.54 & 0.25 \\
\hline $\mathrm{Al}_{2} \mathrm{O}_{3}$ & 15.01 & 15.16 & 15.03 & 15.34 & 14.5 & 14.77 & 14.98 & 14.5 & 15.01 \\
\hline $\mathrm{Fe}_{2} \mathrm{O}_{3}$ & 2.82 & 3.06 & 2.51 & 3.06 & 3.01 & 2.98 & 2.19 & 2.64 & 3.02 \\
\hline $\mathrm{MnO}$ & 0.03 & 0.02 & 0.04 & 0.02 & 0.03 & 0.03 & 0.02 & 0.03 & 0.08 \\
\hline $\mathrm{MgO}$ & 1.03 & 1.12 & 1.06 & 1.3 & 0.86 & 1.35 & 0.94 & 1.22 & 1.31 \\
\hline $\mathrm{Na}_{2} \mathrm{O}$ & 5.68 & 5.95 & 5.19 & 5.61 & 5.5 & 5.24 & 5.73 & 5.52 & 5.26 \\
\hline $\mathrm{K}_{2} \mathrm{O}$ & 2.21 & 2.31 & 2.54 & 2.63 & 2.22 & 2.52 & 2.48 & 2.35 & 2.57 \\
\hline $\mathrm{P}_{2} \mathrm{O}_{5}$ & 0.09 & 0.15 & 0.21 & 0.2 & 0.16 & 0.13 & 0.1 & 0.26 & 0.11 \\
\hline LOI & 0.42 & 0.6 & 0.61 & 1.07 & 0.63 & 0.84 & 0.61 & 0.78 & 0.68 \\
\hline TOTAL & 99.9 & 100.9 & 100.1 & 100.1 & 99.89 & 100.57 & 100.18 & 99.32 & 100.05 \\
\hline $\mathrm{K}_{2} \mathrm{O} / \mathrm{Na}_{2} \mathrm{O}$ & 0.39 & 0.39 & 0.49 & 0.47 & 0.40 & 0.48 & 0.43 & 0.43 & 0.49 \\
\hline $\mathrm{CaO} / \mathrm{Na}_{2} \mathrm{O}$ & 0.38 & 0.38 & 0.41 & 0.42 & 0.37 & 0.40 & 0.38 & 0.29 & 0.40 \\
\hline $\mathrm{A} / \mathrm{CNK}$ & 1.13 & 1.09 & 1.21 & 1.14 & 1.14 & 1.19 & 1.12 & 1.21 & 1.2 \\
\hline $\mathrm{A} / \mathrm{NK}$ & 1.28 & 1.23 & 1.33 & 1.27 & 1.27 & 1.3 & 1.24 & 1.25 & 1.31 \\
\hline $\mathrm{Ba}$ & 963 & 944 & 847 & 433 & 795 & 638 & 480 & 537 & 939 \\
\hline $\mathrm{Rb}$ & 62 & 61 & 37 & 54 & 47 & 49 & 63 & 49 & 45 \\
\hline $\mathrm{Sr}$ & 310 & 640 & 450 & 305 & 335 & 710 & 330 & 586 & 430 \\
\hline $\mathrm{Zr}$ & 69 & 153 & 93 & 95 & 76 & 76 & 165 & 87 & 135 \\
\hline $\mathrm{Nb}$ & 2.15 & 2.34 & 0.57 & 3.4 & 2.77 & 2.76 & 2.42 & 3.68 & 0.55 \\
\hline $\mathrm{Ni}$ & 16 & 8 & 7 & 16 & 29 & 24 & 30 & 9 & 13 \\
\hline $\mathrm{Co}$ & 1.8 & 0.9 & 3.8 & 4 & 1.6 & 1.1 & 2.2 & 1.2 & 0.5 \\
\hline $\mathrm{Zn}$ & 21 & 47 & 22 & 54 & 21 & 35 & 23 & 13 & 22 \\
\hline $\mathrm{Cr}$ & 50 & 115 & 39 & 12 & 125 & 84 & 101 & 115 & 278 \\
\hline $\mathrm{Y}$ & 11 & 5 & 4 & 11 & 9 & 9 & 8 & 9 & 6 \\
\hline Cs & 0.71 & 0.49 & 0.37 & 0.71 & 0.76 & 0.91 & 0.58 & 0.38 & 0.48 \\
\hline $\mathrm{Ta}$ & 0.73 & 2.12 & 3.06 & 5.46 & 0.68 & 0.05 & 1.21 & 0.05 & 0.04 \\
\hline Hf & 2.34 & 4.23 & 4.21 & 3.75 & 3.67 & 3.06 & 3.06 & 3.48 & 3.73 \\
\hline Th & 1 & 2 & 3 & 2.7 & 1 & 5 & 3 & 4 & 2 \\
\hline $\mathrm{Ga}$ & 2.51 & 2.03 & 4.96 & 6.23 & 3.67 & 5.01 & 4.06 & 2.34 & 7.56 \\
\hline $\mathrm{La}$ & 10.6 & 9.7 & 11.1 & 10.2 & 10.5 & 11.1 & 9.8 & 9.8 & 10.6 \\
\hline $\mathrm{Ce}$ & 30.3 & 26.59 & 26.6 & 28.1 & 26.8 & 22.3 & 20.7 & 31.2 & 26.65 \\
\hline $\operatorname{Pr}$ & 9 & 19 & 66 & 48 & 43 & 32 & 28 & 19 & 38 \\
\hline $\mathrm{Nd}$ & 13.1 & 10.3 & 12.3 & 9.6 & 11 & 9.7 & 11.4 & 12.5 & 12.3 \\
\hline $\mathrm{Sm}$ & 2.1 & 1.6 & 2.1 & 1.1 & 1.2 & 1.5 & 1.5 & 2.3 & 1 \\
\hline $\mathrm{Eu}$ & 0.7 & 0.7 & 0.4 & 0.7 & 0.7 & 0.5 & 0.7 & 0.6 & 0.6 \\
\hline Gd & 1.5 & 2.2 & 1.1 & 2 & 0.5 & 2 & 0.7 & 0.61 & 1.7 \\
\hline $\mathrm{Tb}$ & 0.1 & 0.2 & 0.1 & 0.3 & 0.2 & 0.1 & 0.3 & 0.1 & 0.3 \\
\hline Dy & 0.9 & 1.3 & 0.9 & 1.3 & 0.8 & 1.5 & 1.5 & 0.9 & 0.5 \\
\hline Но & 0.3 & 0.1 & 0.2 & 0.2 & 0.1 & 0.1 & 0.3 & 0.2 & 0.1 \\
\hline Er & 0.5 & 0.2 & 0.3 & 0.1 & 0.15 & 0.8 & 0.2 & 0.7 & 0.2 \\
\hline Tm & 0.1 & 0.1 & 0.1 & 0.1 & 0.1 & 0.1 & 0.1 & 0.1 & 0.1 \\
\hline $\mathrm{Yb}$ & 0.3 & 0.3 & 0.4 & 0.7 & 0.7 & 0.7 & 0.4 & 0.5 & 0.5 \\
\hline $\mathrm{Lu}$ & 0.1 & 0.1 & 0.1 & 0.1 & 0.1 & 0.3 & 0.1 & 0.1 & 0.1 \\
\hline $\mathrm{Eu} / \mathrm{Eu}^{*}$ & 1.14 & 1.21 & 1.44 & 1.16 & 2.76 & 1.06 & 2.09 & 1.55 & 1.41 \\
\hline$(\mathrm{La} / \mathrm{Yb}) \mathrm{N}$ & 23.85 & 21.82 & 18.73 & 9.84 & 10.12 & 10.7 & 16.54 & 13.23 & 14.31 \\
\hline
\end{tabular}

Table 2. CIPW normative compositions for the Ngovayang trondhjemitic orthogneisses.

\begin{tabular}{|c|c|c|c|c|c|c|c|c|c|}
\hline & \multicolumn{9}{|c|}{ Amphibole-Garnet Orthogneiss } \\
\hline & GAG01 & GAG02 & GAG03 & GAG04 & GAG05 & GAG06 & GAG07 & GAG08 & GAG09 \\
\hline Quartz & 21.80 & 17.19 & 26.52 & 25.46 & 25.23 & 21.85 & 27.06 & 24.74 & 27.34 \\
\hline Corundum & 0.00 & 0.00 & 0.00 & 0.29 & 0.24 & 0.00 & 0.30 & 0.00 & 0.16 \\
\hline Orthoclase & 13.30 & 18.32 & 14.72 & 16.25 & 12.17 & 16.31 & 14.07 & 16.37 & 13.95 \\
\hline Albite & 49.08 & 54.75 & 44.68 & 45.78 & 46.46 & 47.72 & 42.90 & 41.80 & 42.73 \\
\hline Anorthite & 7.57 & 0.63 & 7.34 & 5.67 & 8.26 & 5.33 & 9.29 & 10.68 & 9.76 \\
\hline Diopside & 1.14 & 5.05 & 0.00 & 0.00 & 0.00 & 2.02 & 0.00 & 0.00 & 0.00 \\
\hline Ilmenite & 0.17 & 0.17 & 0.11 & 0.19 & 0.09 & 0.04 & 0.11 & 0.02 & 0.04 \\
\hline Magnetite & 0.46 & 0.49 & 0.34 & 0.34 & 0.50 & 0.44 & 0.42 & 0.41 & 0.43 \\
\hline Zircon & 0.01 & 0.02 & 0.03 & 0.02 & 0.02 & 0.02 & 0.02 & 0.01 & 0.03 \\
\hline
\end{tabular}




\begin{tabular}{llllllllll}
\hline & \multicolumn{2}{l}{ Amphibole-Garnet Orthogneiss } & & & \\
\cline { 2 - 10 } & GAG01 & GAG02 & GAG03 & GAG04 & GAG05 & GAG06 & GAG07 & GAG08 & GAG09 \\
\hline Chromite & 0.01 & 0.02 & 0.02 & 0.00 & 0.01 & 0.01 & 0.02 & 0.01 & 0.03 \\
Apatite & 0.45 & 0.12 & 0.40 & 0.50 & 0.26 & 0.24 & 0.36 & 0.17 & 0.17 \\
Sum & 99.42 & 100.3 & 98.33 & 99.06 & 99.43 & 98.92 & 99.73 & 99.38 & 99.73 \\
$\mathrm{~T}^{\circ}$ Liquidus $\left({ }^{\circ} \mathrm{C}\right)$ & 840 & 842 & 799 & 807 & 824 & 828 & 817 & 831 & 811 \\
Magma density $(\mathrm{Kb} / \mathrm{m} 3)$ & 2420 & 2410 & 2400 & 2400 & 2420 & 2410 & 2410 & 2410 & 2410 \\
\hline
\end{tabular}

Table 2. Continued.

\begin{tabular}{|c|c|c|c|c|c|c|c|c|c|}
\hline & \multicolumn{5}{|c|}{ Biotite - Pyroxene Orthogneiss } & \multicolumn{4}{|c|}{ Pyroxene Orthogneiss } \\
\hline & GPB01 & GPB02 & GPB03 & GPB04 & GPB05 & GAP01 & GAP02 & GAP03 & GAP04 \\
\hline Quartz & 23.04 & 21.06 & 24.97 & 25.19 & 19.30 & 24.11 & 22.71 & 24.21 & 23.06 \\
\hline Corundum & 0.00 & 0.00 & 0.35 & 0.00 & 0.00 & 0.00 & 0.00 & 0.55 & 0.00 \\
\hline Orthoclase & 13.06 & 13.65 & 15.01 & 13.12 & 15.54 & 14.89 & 14.66 & 13.89 & 15.19 \\
\hline Albite & 48.06 & 50.35 & 43.92 & 46.54 & 47.47 & 44.34 & 48.49 & 46.71 & 44.51 \\
\hline Anorthite & 8.93 & 7.84 & 9.25 & 8.32 & 8.91 & 9.34 & 7.83 & 6.34 & 9.75 \\
\hline Diopside & 0.48 & 1.54 & 0.00 & 0.00 & 0.00 & 0.00 & 1.31 & 0.00 & 0.00 \\
\hline Ilmenite & 0.06 & 0.04 & 0.09 & 0.06 & 0.04 & 0.06 & 0.04 & 0.06 & 0.17 \\
\hline Magnetite & 0.49 & 0.53 & 0.44 & 0.54 & 0.53 & 0.52 & 0.38 & 0.47 & 0.53 \\
\hline Zircon & 0.01 & 0.03 & 0.02 & 0.02 & 0.02 & 0.02 & 0.03 & 0.02 & 0.03 \\
\hline Chromite & 0.01 & 0.02 & 0.01 & 0.00 & 0.03 & 0.02 & 0.02 & 0.03 & 0.06 \\
\hline Apatite & 0.21 & 0.36 & 0.50 & 0.38 & 0.47 & 0.31 & 0.24 & 0.62 & 0.26 \\
\hline Sum & 99.49 & 100.3 & 99.52 & 99.28 & 99.09 & 99.75 & 99.58 & 98.56 & 99.39 \\
\hline $\mathrm{T}^{\circ}$ Liquidus $\left({ }^{\circ} \mathrm{C}\right)$ & 835 & 849 & 832 & 868 & 823 & 837 & 826 & 830 & 845 \\
\hline Magma density $(\mathrm{Kb} / \mathrm{m} 3)$ & 2420 & 2420 & 2410 & 2430 & 2410 & 2420 & 2410 & 2410 & 2420 \\
\hline
\end{tabular}

\section{Results}

\subsection{Petrography}

Field observations revealed that, the Ngovayang massif consists of migmatitic gneisses and granitoids, intruded by basalt or pyroxene tonalite which are interlayered with garnet magnetite quartzite (Figure 1a, [1]. Most of the rock types [4, $19,1]$, found at neighbourhood, are present in the Ngovayang massif as well.

The Ngovayang trondhjemitic gneisses outcrop under plate and boulder types (Figure 2a and Figure 2b). They are fine to medium grained and display light to dark-grey colour. They feature a millimetre to centimetre scale veins (Figure 2a), which are composed mainly by leucocratic minerals (quartz and plagioclase). These veins cross-cut or are parallel to foliation marked by alternating layers of ferromagnesian (amphibole, biotite, pyroxene) and felsic minerals. The Ngovayang trondhjemitic gneisses can be differentiated by their texture and mineral proportions. Indeed we identified amphibole-garnet bearing trondhjemitic gneiss, biotite bearing trondhjemitic gneiss and biotite-orthopyroxene bearing trondhjemitic gneiss. Major rock-forming minerals of these gneisses are quartz (25-35 vol. \%), plagioclase (3540 vol. \%), K-feldspath ( $<5$ vol. \%), hypersthene and biotite (10-15 vol. \%); sometimes the presence of garnet and amphibole $(<5-10$ vol. \%) were observed. Retromorphic sericite (Figure 2c), epidote and chlorite are always present. Quartz occurs in the matrix and display ondulose extinction (Fig. 2f) and sometimes as inclusion minerals biotite or orthopyroxene (Figure 2e). We noticed new elongate grains which growing of quartz crystal (Figure 2f). Opaque minerals are present surrounding or inclusion within biotite crystals (Figure 2d). Accessory minerals include zircon, apatite, ilmenite, magnetite, titanite and monazite.

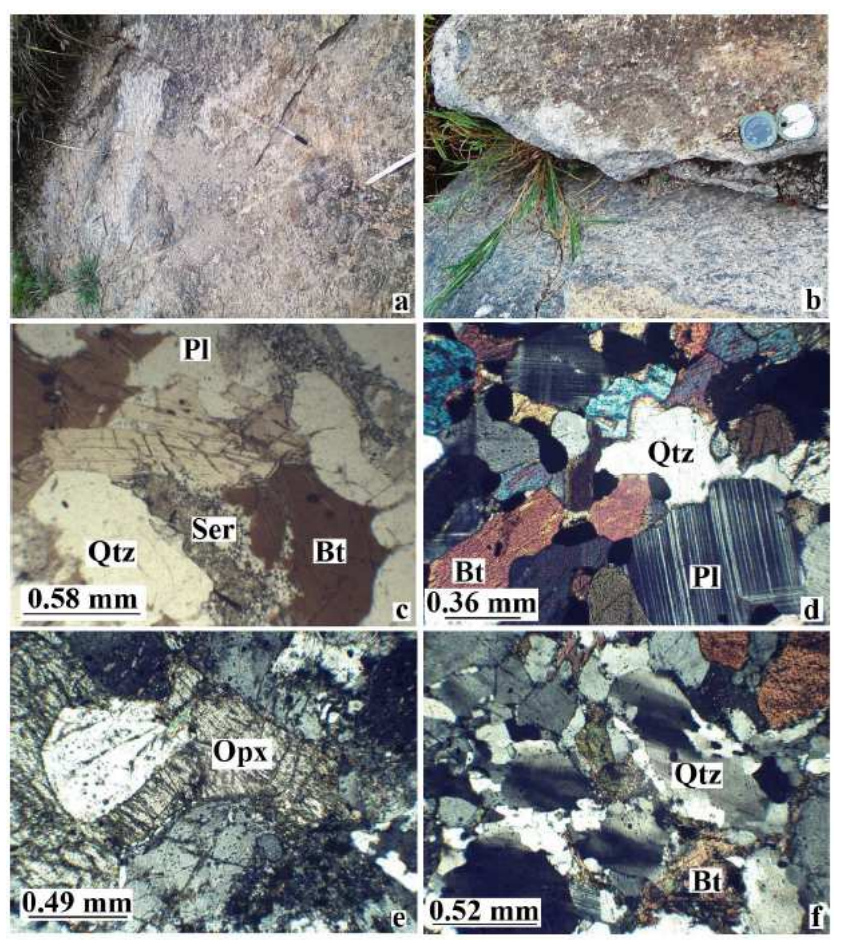

Figure 2. $a$ - and b-Some outcrops atop Ngovayang trondhjemitic gneisses; $c$ - $d$ - - - and f-Microphotographs showing mineral textures relationship in the some Ngovayang trondhjemitic gneisses.

\subsection{Whole-rock Geochemistry}

\subsubsection{Major Elements}

In the An-Ab-Or diagram [35], all the Ngovayang samples plot in the trondhjemite field (Figure 3a) and define a 
trondhjemite trend in the ternary K-Na-Ca diagram (Figure 3b, [36]. These trondhjemitic gneisses belong to calc alkaline serie (Figure 3c; [37], and their plots fall in the I-type field (Figure 3e; [38], respectively. All samples fall in the peraluminous field (Figure 3d; [39] and range from weakly Itype to S-type with nearly constant $\mathrm{A} / \mathrm{CNK}$ values between 1.04 and 1.24 (table 1 and Figure 3d). These samples are silicic $\left(\mathrm{SiO}_{2}=68.08-71.76 \%\right.$, table 1) and plot in the high silicate adakite (HAS) field in the $\mathrm{MgO}$ vs. $\mathrm{SiO}_{2}$ diagram (Figure 3f; [40]. According to the $\mathrm{CaO} / \mathrm{Na}_{2} \mathrm{O}$ ratio, gneisses of the Ngovayang massif display high values (0.25 0.46). Kpoor $\left(\mathrm{K}_{2} \mathrm{O} / \mathrm{Na}_{2} \mathrm{O}=0.38\right.$ 0.56), and with $\mathrm{Fe}$ strongly prevailing over $\mathrm{Mg}\left(\mathrm{Fe}_{2} \mathrm{O}_{3}(\mathrm{t})=1.943 .06 \mathrm{wt} \%, \mathrm{MgO}=0.86\right.$ 1.43 wt. $\%$ and $\mathrm{Mg}^{\#}=0.220 .34$ table 1). At given $\mathrm{SiO}_{2}$ content, $\mathrm{CaO}$ (1.42 2.34 wt. \%) and $\mathrm{Al}_{2} \mathrm{O}_{3}$ (14.07 15.34 wt. \%) are rather high. The Harker variation diagram (Figure 4) shows a slightly negative $\mathrm{SiO}_{2}$ correlation and $\mathrm{Fe}_{2} \mathrm{O}_{3}(\mathrm{t})$, $\mathrm{Al}_{2} \mathrm{O}_{3}, \mathrm{MgO}, \mathrm{CaO}$ and $\mathrm{Na}_{2} \mathrm{O}$ and a positive correlation with $\mathrm{MnO} . \mathrm{SiO}_{2}$ vs. $\mathrm{P}_{2} \mathrm{O}_{5}$ binary diagram shows a subvertical trend (Figure 4). The CIPW normative composition of these samples displays two normative assemblages defined by the presence or absence of diopside and corundum: quartzdiopside-hypersthene assemblage (GAG01, GAG02, GAG03, GAG06, GAG08, GPB01, GPN02, GPB04, GPB05, GAP01, GAP02 and GAP04) and quartz-corundumhypersthene assemblage (GAG04, GAG05, GAG07, GAG09, GPB03 and GAP03), (table 2).
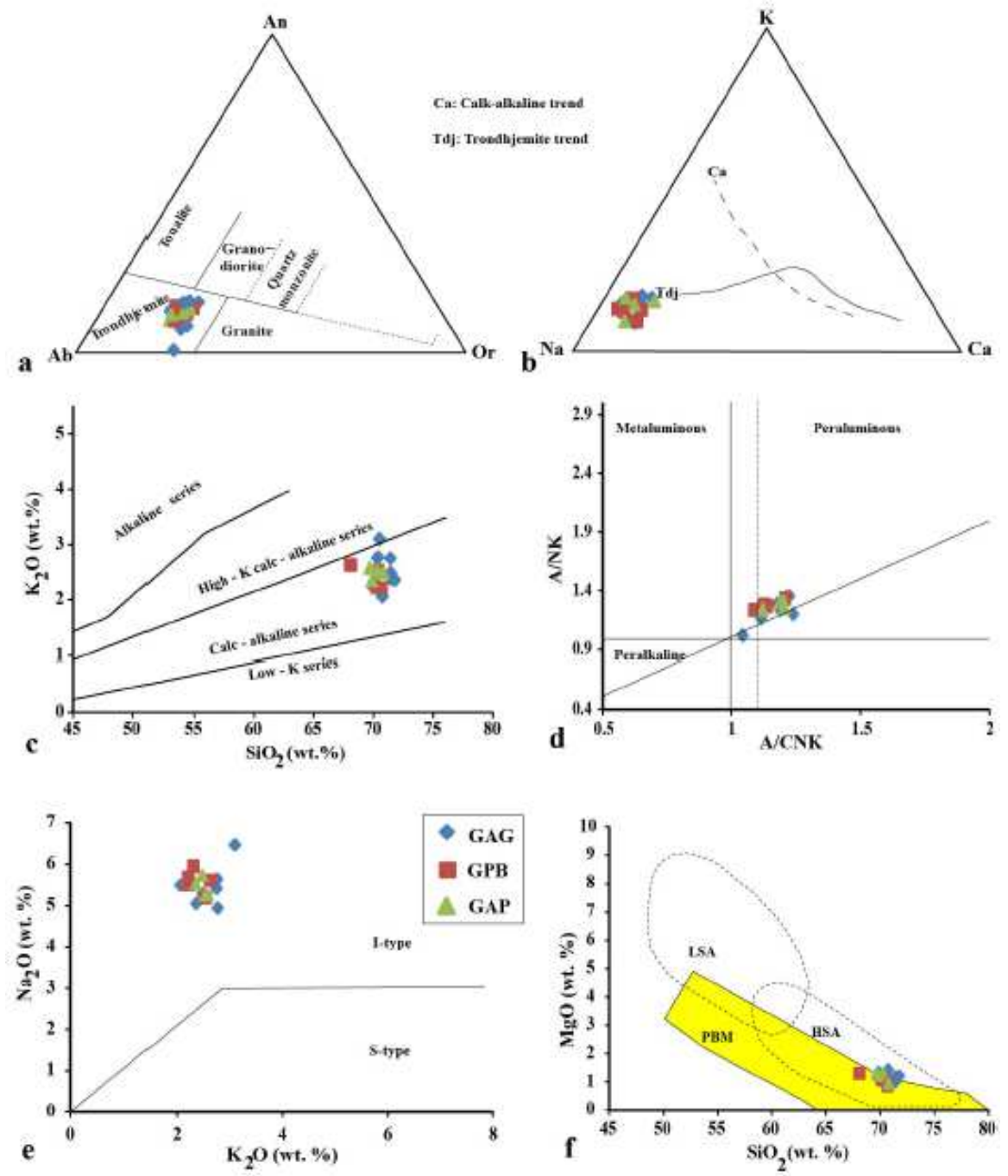

Figure 3. Ternary diagrams: a-Ab-Or-An [35] and b-Na-Ca-K [36]; binary diagrams: c- $\mathrm{K}_{2} \mathrm{O}$ vs. $\mathrm{SiO}_{2}$ [37]; $d-\mathrm{A} / \mathrm{NK}\left(\mathrm{molar}_{\mathrm{ratio}} \mathrm{Al}_{2} \mathrm{O}_{3} /\left(\mathrm{Na}_{2} \mathrm{O}+\mathrm{K}_{2} \mathrm{O}\right)\right.$ vs A/CNK (molar ratio $\mathrm{Al}_{2} \mathrm{O}_{3} /\left(\mathrm{CaO}+\mathrm{Na}_{2} \mathrm{O}+\mathrm{K}_{2} \mathrm{O}\right.$, [39] e- $\mathrm{Na}_{2} \mathrm{O}$ vs. $\mathrm{K}_{2} \mathrm{O}$ diagram [38] and f-MgO-SiO $\mathrm{O}_{2}(\mathrm{PMB}=$ experimentally derived partial melts from metabasaltic rocks. LSA=low silica adakite. HAS=high silica adakite after [40].

\subsubsection{Trace Elements}

$\mathrm{Rb}$ content varies from 29 to $63 \mathrm{ppm}$. Gneisses are very low in $\mathrm{Th}(18 \mathrm{ppm})$ and are rich in $\mathrm{Sr}(308710 \mathrm{ppm})$ and $\mathrm{Ba}$ (302 $1040 \mathrm{ppm}$ ) respectively. These gneisses display low $(\mathrm{La} / \mathrm{Yb})_{\mathrm{N}}$ of 9.8423 .85 with slightly positive Eu anomaly
$(\mathrm{Eu} / \mathrm{Eu}=0.966$ 2.760). The spidergrams of Ngovayang gneisses samples show distinctive $\mathrm{Pr}$ positive anomalies (Figure 5a and Figure 5b) and negative anomaly in $\mathrm{Nb}$ (Fig. $5 b)$. All these spidergrams display depletion in earth heavy (Figure 5a and Figure 5b). 


\subsubsection{Isotopic Analyses}

Ten whole rock samples were analysed for ${ }^{147} \mathrm{Sm}-{ }^{143} \mathrm{Nd}$ isotopic compositions such as GAG01, GAG02, GAG03 and GAG08 for amphibole-garnet gneiss; GPB01, GPB02 and GPB05 for biotite-pyroxene gneiss and GAP01, GAP02 and GAP03 for pyroxene gneiss. Six whole rock samples were studied for Rb-Sr isotopic compositions including GAG02, GAG03 and GAG08 for amphibole-garnet gneiss; GPB02
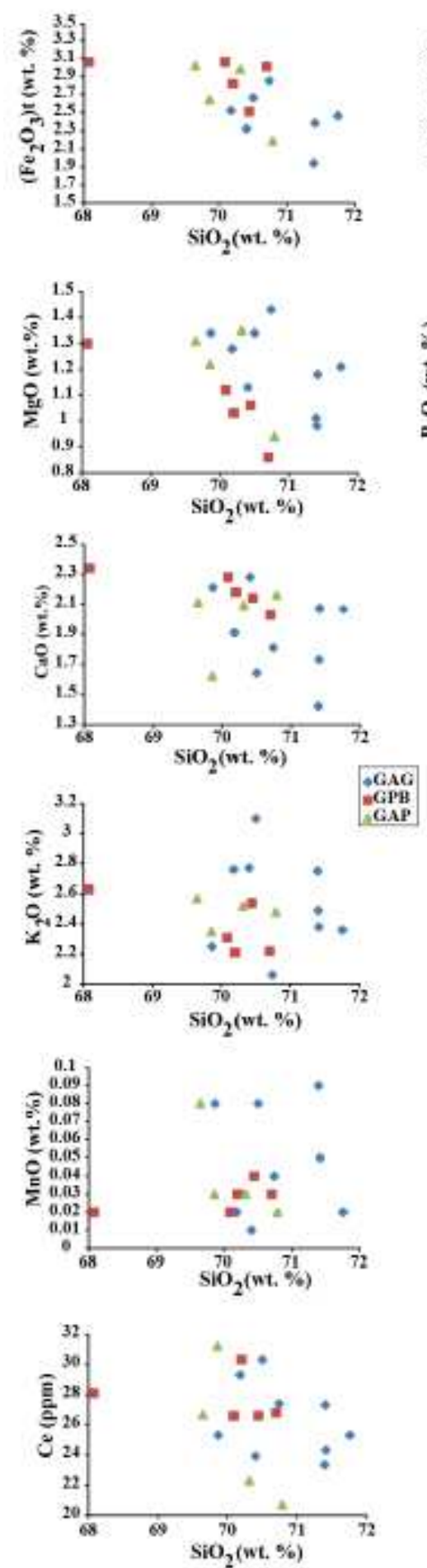

for biotite-pyroxene gneiss and GAP01, GAP02 for pyroxene gneiss. The gneisses of the Ngovayang massif have ${ }^{87} \mathrm{Sr} /{ }^{86} \mathrm{Sr}$ ratio values between $0.70367-0.74191$ and the initial ${ }^{87} \mathrm{Sr}{ }^{86} \mathrm{Sr}$ ratio value range from 0.69308 to 0.72496 (table 3). Their ${ }^{143} \mathrm{Nd} /{ }^{144} \mathrm{Nd}$ ratio values vary between $0.51112-0.51148$ with initial ${ }^{143} \mathrm{Nd} /{ }^{144} \mathrm{Nd}$ isotopic values between 0.51102 and 0.51179 and a negative $\mathrm{Nd}(\mathrm{T})$ values $(-1.43$ to -16.48 , table $3)$.
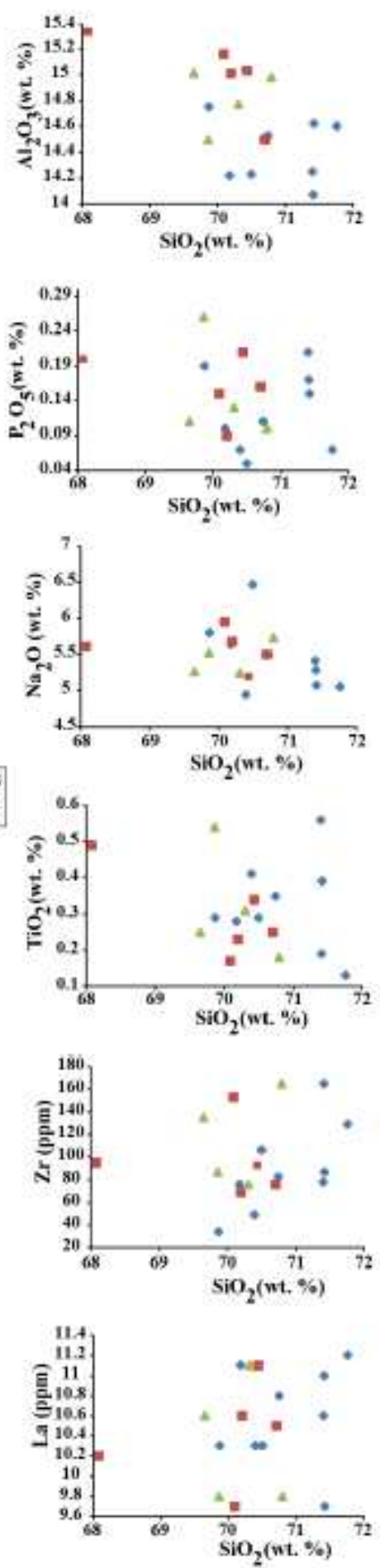

Figure 4. Binary diagrams of $\mathrm{SiO}_{2}$ vs. major-element oxides (wt. \%) and some trace elements (ppm) for Ngovayang orthogneisses. 


\subsection{Thermometry}

\subsubsection{Zircon}

Based on zircon thermometry, temperatures of Ngovayang orthogneisses range between 653 and $782^{\circ} \mathrm{C}$ (aver. $729^{\circ} \mathrm{C}$ ). These values correspond to those obtained in the amphibolite garnet gneiss but with a different average temperature value (aver. $722^{\circ} \mathrm{C}$ ). In the biotite pyroxene gneiss, temperatures correspond to an interval range from 710 to $765^{\circ} \mathrm{C}$ (aver. $730^{\circ} \mathrm{C}$ ), whereas, they vary between 717 and $775^{\circ} \mathrm{C}$ (aver. $746^{\circ} \mathrm{C}$ ) for the pyroxene gneiss (table 4$)$.
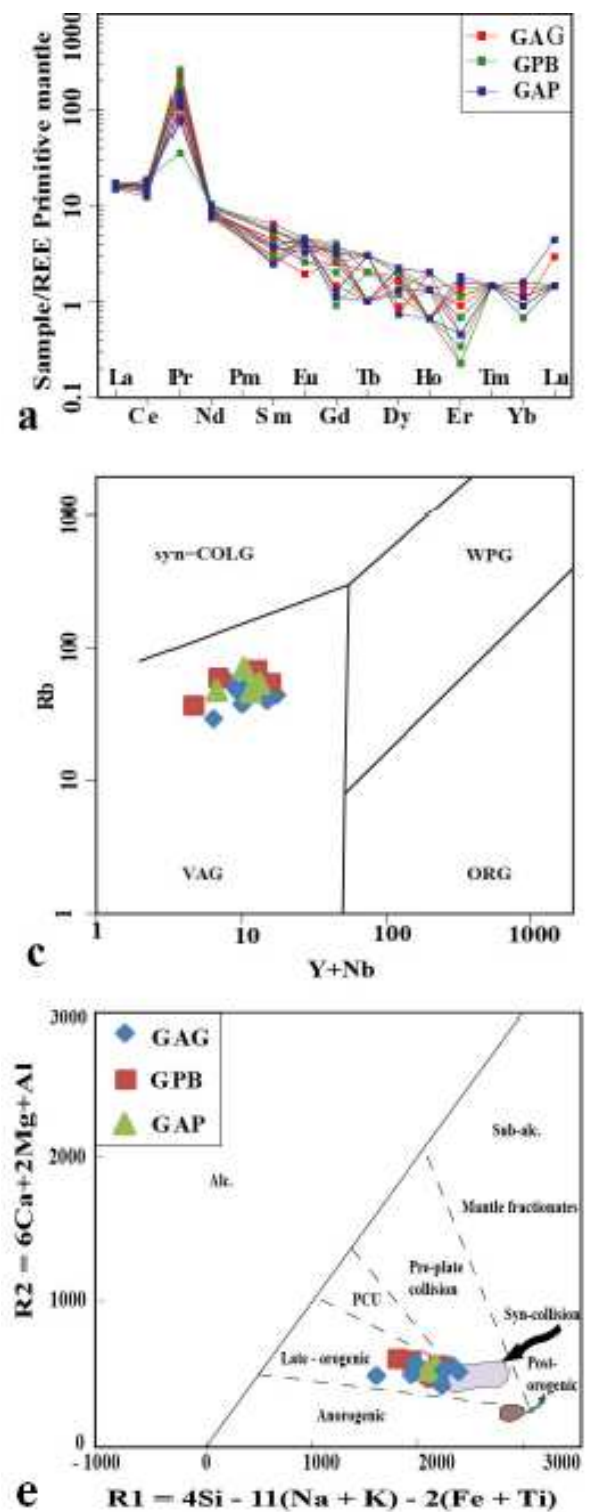

\subsubsection{Monazite}

The monazite thermometry gives us temperatures of Ngovayang gneisses varying from 678 to $723^{\circ} \mathrm{C}$ (aver. $706^{\circ} \mathrm{C}$ ). Temperatures are $678-718^{\circ} \mathrm{C}$ in the amphibolite garnet gneiss with an average value of $705^{\circ} \mathrm{C}$. In the biotite pyroxene gneiss, temperatures correspond to an interval range between 695 and $718^{\circ} \mathrm{C}$ (aver. $706^{\circ} \mathrm{C}$ ), whereas they range between 692 and $723^{\circ} \mathrm{C}$ (aver. $708^{\circ} \mathrm{C}$ ) for the pyroxene gneiss (table 4).
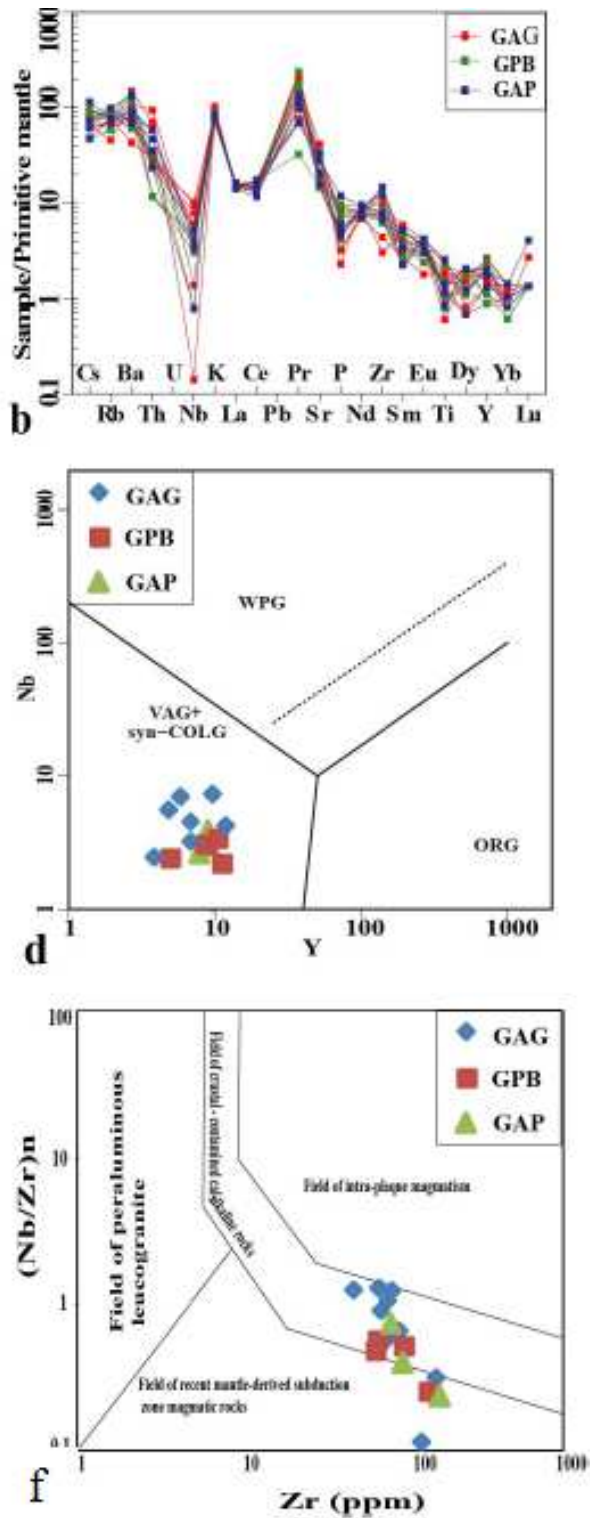

Figure 5. Binary plots: a-Chondrite-normalized REE patterns [65], d-Primitive mantle-normalized trace element spider diagrams [65], cRb vs. Y+Nb (VAG, volcanic arc granite; syn-COLG syn-collision granite; WPG within plate granite; ORG oceanic ridge granite; [41]; d- Nb vs. Y [41]; e-Multication diagram R2 vs R1 (Alc., alcalin; Sub-alc. Sub-alcalin and PCU, Post collision Uplift; [66] and f-(Nb/Zr)n ratio vs. Zr.

\subsubsection{Apatite}

According to apatite thermometry, temperatures of Ngovayang orthogneisses range between 671 and $798^{\circ} \mathrm{C}$ (aver. $738^{\circ} \mathrm{C}$ ). Temperatures are $671773^{\circ} \mathrm{C}$ in the amphibolites garnet gneiss with an average value of $724^{\circ} \mathrm{C}$. In the biotite pyroxene gneiss, temperatures correspond to an interval range between 713 and $797^{\circ} \mathrm{C}$ (aver. $757^{\circ} \mathrm{C}$ ), whereas they vary between 716 and $798^{\circ} \mathrm{C}$ (aver. $746^{\circ} \mathrm{C}$ ) for the pyroxene gneiss (table 4 ). 
Table 3. Rb and Sr abundances and isotopic composition of Sm/Nd ratios, $\varepsilon N d$ and $T_{D M}$ from some Ngovayang trondhjemitic gneiss samples.

\begin{tabular}{|c|c|c|c|c|c|c|c|c|c|}
\hline & Sample & Sm & Nd & ${ }^{147} \mathrm{Sm} /{ }^{144} \mathrm{Nd}$ & ${ }^{143} \mathrm{Nd} /{ }^{144} \mathrm{Nd} \pm 2 \sigma_{\mathrm{m}}$ & $(\mathrm{RNd}) \mathrm{i}$ & $\varepsilon N d(0)$ & $\varepsilon N d(T)$ & $\mathrm{T}_{\mathrm{DM}}(\mathrm{Ma})$ \\
\hline \multirow{4}{*}{$\begin{array}{l}\text { Amp-Grt bearing } \\
\text { orthogneiss }\end{array}$} & GAG01 & 5.541 & 24.14 & 0.092 & $0.511258(4)$ & 0.51113 & -26.88 & -14.33 & 2361 \\
\hline & GAG02 & 5.982 & 35.10 & 0.099 & $0.511325(6)$ & 0.51119 & -25.57 & -13.21 & 2416 \\
\hline & GAG03 & 4.892 & 27.26 & 0.107 & $0.511469(6)$ & 0.51132 & -22.76 & -10.61 & 2392 \\
\hline & GAG08 & 2.174 & 10.90 & 0.139 & $0.511983(4)$ & 0.51179 & -12.74 & -1.43 & 2373 \\
\hline \multirow{3}{*}{$\begin{array}{l}\text { Bt-Opx bearing } \\
\text { orthogneiss }\end{array}$} & GAP01 & 2.008 & 6.503 & 0.096 & $0.511252(4)$ & 0.51112 & -27 & -14.55 & 2448 \\
\hline & GAP02 & 2.634 & 14.44 & 0.105 & $0.511403(5)$ & 0.51126 & -24.05 & -11.9 & 2440 \\
\hline & GAP03 & 4.854 & 24.43 & 0.105 & $0.511466(4)$ & 0.51132 & -22.82 & -10.6 & 2353 \\
\hline \multirow{2}{*}{$\begin{array}{l}\text { Opx bearing } \\
\text { orthogneiss }\end{array}$} & GPB02 & 3.012 & 17.84 & 0.091 & $0.511277(7)$ & 0.51115 & -26.51 & -13.99 & 2318 \\
\hline & GPB05 & 2.544 & 13.44 & 0.084 & $0.511137(5)$ & 0.51102 & -29.24 & -16.48 & 2357 \\
\hline
\end{tabular}

Table 3. Continued.

\begin{tabular}{|c|c|c|c|c|c|c|c|c|}
\hline & Sample & $\mathbf{R b}(\mathbf{p p m})$ & $\mathrm{Sr}(\mathrm{ppm})$ & ${ }^{87} \mathrm{Rb} /{ }^{86} \mathrm{Sr}$ & ${ }^{87} \mathrm{Sr} /{ }^{86} \mathrm{Sr} \pm 2 \sigma_{\mathrm{m}}$ & (RSr)i & $\varepsilon \operatorname{Sr}(0)$ & $\varepsilon \mathrm{Sr}(\mathrm{T})$ \\
\hline \multirow{3}{*}{ Amp-Grt bearing orthogneiss } & GAG02 & 41 & 767 & 0.15 & $0.728167(7)$ & 0.72363 & 335.94 & 306.97 \\
\hline & GAG03 & 37 & 314 & 0.34 & $0.716411(10)$ & 0.70612 & 169.07 & 57.60 \\
\hline & GAG08 & 41 & 341 & 0.35 & $0.703677(8)$ & 0.69308 & -11.68 & 128.09 \\
\hline Bt-Opx bearing orthogneiss & GPB02 & 74 & 817 & 0.26 & $0.724462(7)$ & 0.71659 & 283.35 & 206.77 \\
\hline \multirow{2}{*}{ Opx bearing orthogneiss } & GAP01 & 42 & 718 & 0.17 & $0.707769(9)$ & 0.70262 & 46.402 & 7.804 \\
\hline & GAP02 & 67 & 350 & 0.56 & $0.741911(10)$ & 0.72496 & 531.03 & 325.96 \\
\hline
\end{tabular}

$\mathrm{m}=$ measured isotopic ratios; $\mathrm{i}=$ calculated initial isotopic ratios; $\varepsilon \mathrm{Nd}(\mathrm{T})$ values were calculated using present day $\left({ }^{143} \mathrm{Nd} /{ }^{144} \mathrm{Nd}\right) \mathrm{CHUR}=0.512638$ and

${ }^{147} \mathrm{Sm} /{ }^{144} \mathrm{Nd}$ ) CHUR=0.1967; CHUR= Chondrite Uniform Reservoir; $\lambda=6.54 .10^{-12} \mathrm{a}^{-1}$ (Steiger and Jäger, 1977) 4.3.4. $\mathrm{Al}_{2} \mathrm{O}_{3} / \mathrm{TiO}_{2}$ ratio

$\mathrm{Al}_{2} \mathrm{O}_{3} / \mathrm{TiO}_{2}$ ratio thermometry gives temperatures of Ngovayang orthogneiss between 732 and $928^{\circ} \mathrm{C}$ with an average of $842^{\circ} \mathrm{C}$. Temperatures are $732886^{\circ} \mathrm{C}$ in the amphibolite garnet gneiss with an average value of $847^{\circ} \mathrm{C}$. In the biotite pyroxene gneiss, temperatures vary from 764 to $908^{\circ} \mathrm{C}$ (aver. $832^{\circ} \mathrm{C}$ ), whereas they are ranged between 773 and $929^{\circ} \mathrm{C}$ (aver. $843^{\circ} \mathrm{C}$ ) for the pyroxene gneiss (table 4 ).

\subsubsection{Rutile}

According to rutile thermometry, temperatures of Ngovayang orthogneisses range between 659 and $819^{\circ} \mathrm{C}$ (aver. $740^{\circ} \mathrm{C}$ ). These extreme temperatures were also recorded in the amphibolite garnet gneiss with an average value of $743^{\circ} \mathrm{C}$. In the biotite pyroxene gneiss, temperatures correspond to an interval range between 681 to $795^{\circ} \mathrm{C}$ (aver. $734^{\circ} \mathrm{C}$ ), whereas they vary from 690 to $811^{\circ} \mathrm{C}$ (aver. $742^{\circ} \mathrm{C}$ ) for the pyroxene gneiss (table 4).

\subsubsection{Normative Temperatures}

The normative liquidus temperatures of Ngovayang orthogneisses range between 799 and $868^{\circ} \mathrm{C}$ with a mean of $830^{\circ} \mathrm{C}$. Temperatures are $799-842^{\circ} \mathrm{C}$ in the amphibolite garnet gneiss with an average value of $822^{\circ} \mathrm{C}$. In the biotite pyroxene gneiss, temperatures are $823868^{\circ} \mathrm{C}$ (aver. $841^{\circ} \mathrm{C}$ ), whereas they range between 826 and $845^{\circ} \mathrm{C}$ (aver. $834^{\circ} \mathrm{C}$ ) for the pyroxene gneiss (table 2).

Table 4. Estimate temperatures for the NTG.

\begin{tabular}{llllllllll}
\hline & \multicolumn{2}{l}{ Amphibole-garnet orthogneiss } & & & & \\
\cline { 2 - 9 } & GAG01 & GAG02 & GAG03 & GAG04 & GAG05 & GAG06 & GAG07 & GAG08 & GAG09 \\
\hline $\mathrm{T}\left({ }^{\circ} \mathrm{C}\right) \mathrm{Zr}$ & 653 & 724 & 782 & 722 & 727 & 709 & 731 & 686 & 764 \\
$\mathrm{~T}\left({ }^{\circ} \mathrm{C}\right) \mathrm{P}_{2} \mathrm{O}_{5}$ & 773 & 671 & 749 & 765 & 723 & 721 & 739 & 694 & 684 \\
$\mathrm{~T}\left({ }^{\circ} \mathrm{C}\right) \mathrm{MnZ}$ & 697 & 678 & 715 & 709 & 718 & 697 & 711 & 707 & 718 \\
$\mathrm{~T}\left({ }^{\circ} \mathrm{C}\right) \mathrm{Al} / \mathrm{Ti}$ & 841.1 & 646 & 789.5 & 936.1 & 868.9 & 841.3 & 882.9 & 885.9 & 732.4 \\
$\mathrm{~T}\left({ }^{\circ} \mathrm{C}\right) \mathrm{Rt} 1$ & 736 & 732.5 & 697 & 819.3 & 758.6 & 732.1 & 773.7 & 779.5 & 659.2 \\
& Biotite-pyroxene orthogneiss & & & & Opx bearing orthogneiss & & \\
& GPB01 & GPB02 & GPB03 & GPB05 & GPB04 & GAP01 & GAP02 & GAP03 & GAP04 \\
$\mathrm{T}\left({ }^{\circ} \mathrm{C}\right) \mathrm{Zr}$ & 707 & 765 & 735 & 710 & 733 & 717 & 775 & 730 & 763 \\
$\mathrm{~T}\left({ }^{\circ} \mathrm{C}\right) \mathrm{P}_{2} \mathrm{O}_{5}$ & 713 & 752 & 775 & 751 & 797 & 739 & 716 & 798 & 733 \\
$\mathrm{~T}\left({ }^{\circ} \mathrm{C}\right) \mathrm{MnZ}$ & 715 & 695 & 718 & 706 & 699 & 704 & 692 & 723 & 714 \\
$\mathrm{~T}\left({ }^{\circ} \mathrm{C}\right) \mathrm{Al} / \mathrm{Ti}$ & 806.9 & 764 & 860.3 & 907.5 & 823.1 & 850 & 773.5 & 928.7 & 818.3 \\
$\mathrm{~T}\left({ }^{\circ} \mathrm{C}\right) \mathrm{Rt} 1$ & 713.7 & 681.2 & 757.8 & 795.5 & 722.9 & 744.3 & 690 & 811.6 & 721.4 \\
\hline
\end{tabular}

\subsection{Dating Results}

Ten whole rock samples were analysed for ${ }^{147} \mathrm{Sm}^{-143} \mathrm{Nd}$ isotope compositions. The results are presented in table 3 . The $T_{D M}$ model ages in these gneisses range from 2597 to 2318 Ma. In the amphibole-garnet gneiss, the $T_{D M}$ model ages have an interval varying between 2416 and $2361 \mathrm{Ma}$.
These ages range from 2597 to $2318 \mathrm{Ma}$ in the biotitepyroxene gneiss whereas pyroxene orthogneiss shows 2448 2353 Ma age intervals (table 3).

\section{Discussion}

The trondhjemitic gneisses of the Ngovayang area have a 
magmatic origin (Figure $3 \mathrm{e}$ ) and this observation can also be confirmed by the variability of $\mathrm{CaO} / \mathrm{Fe}_{2} \mathrm{O}_{3}$ (t) ratios (0.61$0.98)$ and low $\mathrm{P}_{2} \mathrm{O}_{5}$ contents $(0.050 .26$ wt. \%; table 1$)$, which are similar to those of I-type granitoids [38]. Indeed, most samples fall in the S-type field (Figure 3d) and some values of (RSr)i are above 0.708 which is characteristic of Stype granitoids (e.g. GAG02, GPB02 and GAP02, table 3). The tectonic discrimination diagrams of [41] are used to determining the tectonic setting. In the binary diagrams, all samples plot in the volcanic arc granite fields (VAG, Figure $5 \mathrm{c}$ and $5 \mathrm{~d}$ ), in spite of the partially peraluminous and S-type characteristics of these trondhjemitic gneisses. Their I- and S- type characters suggest that juvenile materials have been partially remobilized or recycled. These gneisses have slightly to moderate $\mathrm{MgO}$ (table 1), $\mathrm{Cr}$ and $\mathrm{Ni}$ contents (Figure 6e and 6f), suggesting small mantle-source contribution during genesis of their magma. The wide range values of both $\mathrm{Sr}(\mathrm{i})$ ratio and $\mathrm{Nd}(\mathrm{t})$ of the trondhjemitic gneisses (Figure 7 and table 3 ) and high $\mathrm{Sr}$ content indicate an insignificant contribution of mantle in their genesis. These gneisses have evolved ISr $(0.7036770 .741911$, table $3 \mathrm{Nd}(\mathrm{t})$ (from -16.48 to -10.6 , table 3 ), proving assimilation of some upper and lower crustal materials [37, 42-43]. The considerable variations of isotopic data values (Figure 7 and table 3 ) indicate that crustal contamination has played a main role in modifying the primary composition of these trondhjemitic gneisses, representing an old crust probably of Archaean origin [4, 18]. Trondhjemitic gneisses have high $\mathrm{Na}_{2} \mathrm{O}$ and $\mathrm{CaO}$, and slightly positive $\mathrm{Eu}$ anomalies $\left(\mathrm{Eu} / \mathrm{Eu}^{*}\right.$ $=1.02$ 2.8, table 1), which may be due to small amount of Na-rich plagioclase accumulation. Each rock type shows distinct chemical evolution trends and does not display welldefined correlations between many major elements and $\mathrm{SiO}_{2}$ (Figure 3). The presence of garnet and pyroxene which are restite unmixing phase can explain chemical variations that are observed in these gneisses [44]. The interpretations mentioned above show that these chemical variations are mostly inherited from both source and restite unmixing phase. Fractional crystallization and/or assimilation have played a secondary role during their formation. This observation is also confirmed by normative assemblages which display chemical variations (table 2 ). The Ngovayang gneisses have low $\mathrm{K}_{2} \mathrm{O} / \mathrm{Na}_{2} \mathrm{O}$ ratios $(<1$, table 1$)$; slightly positive Eu anomalies (table 1) and they have a trondhjemitic affinity (Figure $3 a$ and $3 b$ ) which is consistent with water present in the melt [45]. Besides it is known that destabilization of plagioclase is caused by both addition of water and the lack of peritectic k-feldspar in the melt source [45]. Hence, the presence of plagioclase in the water melt environment can be explained by slightly positive Eu anomalies and the presence of small amounts of k-feldspar in the source (Figure $3 \mathrm{~b}$, Figure $5 \mathrm{~b}$ ). The $\mathrm{CaO} / \mathrm{Na}_{2} \mathrm{O}$ ratios can be used to highlight the effect of source composition in granitoid melts $[6,34]$. Thus high $\mathrm{CaO} / \mathrm{Na}_{2} \mathrm{O}$ and low $\mathrm{Al}_{2} \mathrm{O}_{3} / \mathrm{TiO}_{2}$ ratio values of trondhjemitic gneisses, could indicative of their origin from the melting of psammite (Figure 6a); however since their $\mathrm{CaO} / \mathrm{Na}_{2} \mathrm{O}$ ratio values are variable and often high (0.25 0.46 , table 1$)$, melts may be originated from metabasaltic, orthogneiss or amphibolite sources, in accordance with laboratory experiments of $[6,34$, 46]. The petrogenetic diagrams of AFM vs CFM and $\mathrm{Rb} / \mathrm{Ba}$ vs $\mathrm{Rb} / \mathrm{Sr}$ indicate that the magmatic source materials are mainly composed of metagreywacke and basalt or igneous rocks (Figure 6b and 6d, respectively). In the $\mathrm{MgO}$ vs $\mathrm{SiO}_{2}$ diagram, all samples plot in the metabasaltic and eclogic field and partially in the melts from both slab and thickened lower crust fields (Figure 6c). Our trondhjemitic gneisses display lower $\mathrm{K}_{2} \mathrm{O} / \mathrm{Na}_{2} \mathrm{O}$ ratio $(0.38-0.51$, table 1) values than those obtained during the partial melting of the thickened lower continental crust with $\mathrm{K}_{2} \mathrm{O} / \mathrm{Na}_{2} \mathrm{O}$ ratios (usually $>0.5$; [47]. Therefore, petrogenesis via thickened lower continental crust can be retained partially for these trondhjemitic gneisses because slight $\mathrm{K}_{2} \mathrm{O} / \mathrm{Na}_{2} \mathrm{O}$ ratios are up to 0.5 (table 1). The lower $\mathrm{K}_{2} \mathrm{O} / \mathrm{Na}_{2} \mathrm{O}$ ratios are consistent with melts from slab melts which display $\mathrm{K}_{2} \mathrm{O} / \mathrm{Na}_{2} \mathrm{O}$ ratios $<$ 0.5 [48-49] and can be mainly retained for these trondhjemitic gneisses. Hence the above interpretations are agreeing with the generation of these trondhjemitic gneiss magmas from both basalt or igneous rocks and from plagioclase-rich sources such as metagreywackes metabasic mixtures, metagreywackes and metabasics with melt-present. The trondhjemitic gneisses have high $\mathrm{K}_{2} \mathrm{O}$ (2.06-3.1 wt. \%) content, low $\mathrm{MgO}\left(0.86-1.43\right.$ wt. \%) content, variable $\mathrm{Mg}^{\#}$ (39.97-54.82) values, Cr (10 $278 \mathrm{ppm})$ content and Ni (536 ppm) values; as well as the obtained calc-alkaline characteristic of magmas (Figure 3c). All these show that magmas derived from the partial melting of both the subducted oceanic slab and lower crust, in agreement with figures 6e and 6f. Moreover, $\mathrm{Sr}$ and $\mathrm{Nd}$ isotope ratios which display a bit variation for GAG01, GAG02, GAG03, GAG08, GPB01, GPB02, GPB05, GAP01, GAP02 and GAP03 (table 2) samples, suggest that the magma of trondhjemitic gneisses share a subduction related magma source [50] and crustal assimilation or heterogeneity in the magma source [51]. Whole rock zircon, monazite, apatite, rutile $[32,34,52]$ and $\mathrm{Al}_{2} \mathrm{O}_{3} / \mathrm{TiO}_{2}$ ratios, [6] can be used to constrain the temperatures of granitoids. Temperatures obtained from these geothermometers are generally very high; however temperatures obtained from zircon, monazite, apatite and rutile are lower than those obtained from $\mathrm{Al}_{2} \mathrm{O}_{3} / \mathrm{TiO}_{2}$ ratios (table 4 ). On the base of experimental studies, accessory minerals (zircon, apatite, monazite and rutile) solubility with zirconium, $\mathrm{P}_{2} \mathrm{O}_{5}, \mathrm{LREE}$ and $\mathrm{TiO}_{2}$ are negatively correlated with $\mathrm{SiO}_{2}$, attesting that they started to crystallize [6, 32, 34, 52-54]. Figure 4 shows that $\mathrm{P}_{2} \mathrm{O}_{5}, \mathrm{Zrn}$ and La solubility are positively correlated with $\mathrm{SiO}_{2}$ in GAG sample contrary to GPB and GAP which display negative trends. All these show that accessory minerals represent a cumulate phase in GAG sample and are inherited from the source in GPB and GAP samples. Hence, temperature constraints within these minerals in GAG sample correspond to evolved phase which extends from 653 to $782{ }^{\circ} \mathrm{C}$ (table 4). In the GPB and GAP samples, we have saturation temperatures $[6,32,34,52-54]$. which yield temperatures 
between 681 and $811^{\circ} \mathrm{C}$ (table 4). During partial melting, concentrations of $\mathrm{Al}_{2} \mathrm{O}_{3}$ in the melt remain constant due to buffering by aluminous minerals (plagioclase and garnet) while the $\mathrm{TiO}_{2}$ concentration increases with increasing temperature due to progressive breakdown of biotite, amphibole, ilmenite and titanite at higher temperatures [6, 55-60]. These gneisses have $\mathrm{Al}_{2} \mathrm{O}_{3}$ with constant values (14.23-15.16, table 1) and temperatures obtained by $\mathrm{Al}_{2} \mathrm{O}_{3} / \mathrm{TiO}_{2}$ ratios are high, indicating that temperatures of crustal anataxis decrease [6]. This probably reflects a decreasing instability of Ti-bearing phases with regressive crustal fusion [6]. These high temperature values are similar to those of normative temperature liquidus (table 2). Hence, they correspond to melting temperatures $\left(732-928^{\circ} \mathrm{C}\right.$, table 4). Each rock type and sample in the same lithology show distinct temperatures since the variable breakdown level of biotite, ilmenite and titanite also produce variable $\mathrm{TiO}_{2}$ accumulation (table 1) at high temperature. The magma densities calculated by temperatures obtained with $\mathrm{Al}_{2} \mathrm{O}_{3} / \mathrm{TiO}_{2}$ thermometer (aver. $2300 \mathrm{~kg} / \mathrm{m}^{3}, 2308 \mathrm{~kg} / \mathrm{m}^{3}$ and
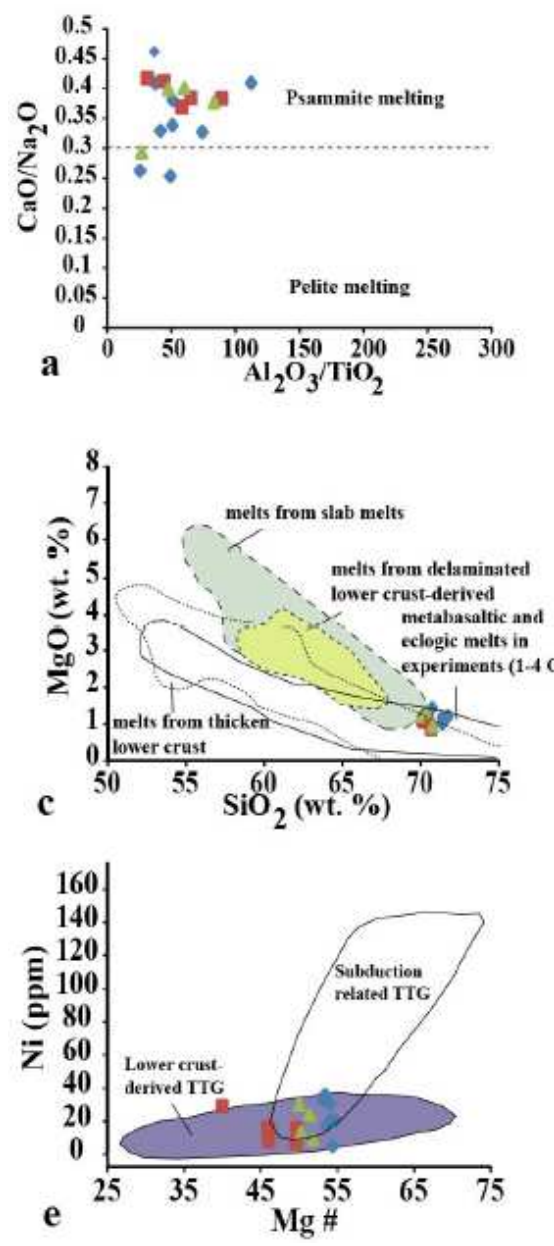

$2306 \mathrm{~kg} / \mathrm{m}^{3}$; table 4) are slightly similar to those obtained by normative composition (aver. $2410 \mathrm{~kg} / \mathrm{m}^{3}, 2418 \mathrm{~kg} / \mathrm{m}^{3}$ and $2415 \mathrm{~kg} / \mathrm{m}^{3}$; table 2). Hence, magma density of these gneisses ranges between 2300 and $2418 \mathrm{~kg} / \mathrm{m}^{3}$.

This study presents Nd-model $\left(\mathrm{T}_{\mathrm{DM}}\right)$ of the Ngovayang gneisses which are distinct. The Ngovayang gneisses have Nd-model ages varying from 2416 to $2392 \mathrm{Ma}, 2597$ to 2318 $\mathrm{Ma}$ and 2448 to $2353 \mathrm{Ma}$ (table 3), in Amp-Grt bearing gneiss, Bt-Opx bearing gneiss and Opx bearing gneiss respectively. Most data show of a Paleoproterozoic model age with only one data displaying a Neoarchean model age (table 3). These old models (Neoarchean and early Paleoproterozoic ages) of trondhjemitic orthogneiss (2597 2318 , table 3) indicate the presence of a component with long crustal residence time in their source. Such a source is compatible with old calc-alkaline arc-rocks. The old age of $2597 \mathrm{Ma}$ obtained in the GPB01 sample and isotopic characters (Figure 7) implies that old Neoarchean crust was part of the Ngovayang in the Nyong Complex [4, 18].
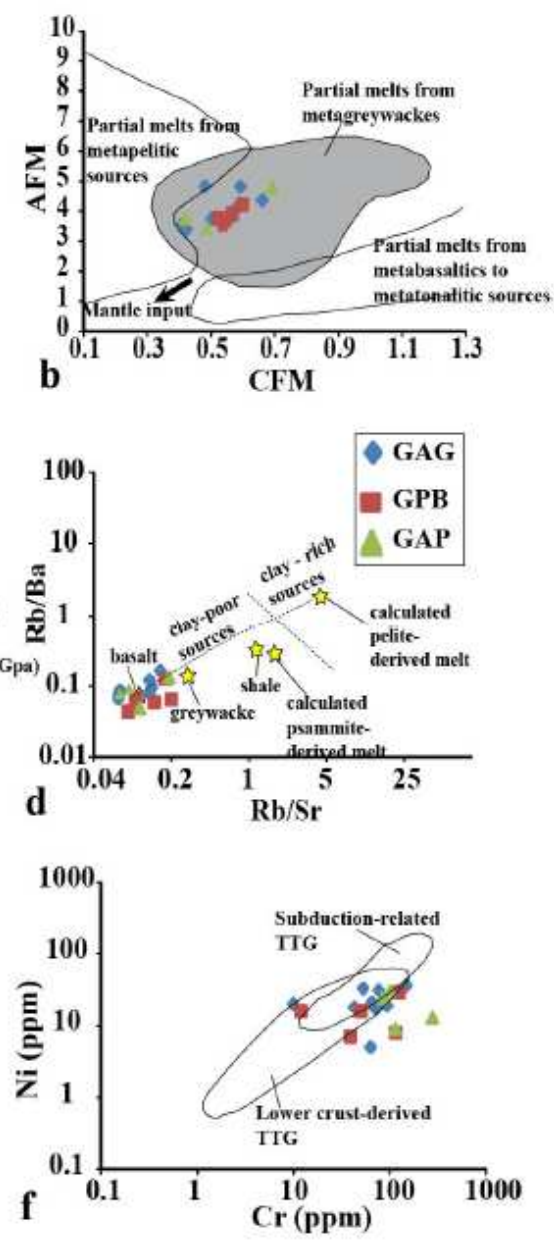

Figure 6. Binary diagrams: a- $\mathrm{CaO} / \mathrm{Na}_{2} \mathrm{O}$ ratio vs. $\mathrm{Al}_{2} \mathrm{O}_{3} / \mathrm{TiO}_{2}$ ratio diagram for trondhjemitic gneisses with boundary between experimental melts derived from metapsammitic And metapelitic sources [34]; b- Molar $\mathrm{Al}_{2} \mathrm{O}_{3} /\left(\left(\mathrm{Fe}_{2} \mathrm{O}_{3}\right) \mathrm{T}+\mathrm{MgO}\right)(\mathrm{AFM})$ vs. molar $\mathrm{CaO} /\left(\left(\mathrm{Fe}{ }_{2} \mathrm{O}_{3}\right) \mathrm{T}+\mathrm{MgO}\right)(\mathrm{CFM})$ diagram (modified after [67]; $\mathrm{c}-\mathrm{MgO}$ vs. $\mathrm{SiO}_{2}$ diagram [67, 68]; $\mathrm{d}$ - $\mathrm{Ni}$ vs $\mathrm{Mg}^{\#}$ diagram [67, 68] and $\mathrm{Ni}$ vs. Cr diagram [67, 68].

In this study, the samples partially plot in the late orogenic field, while, other samples fall into the syn-collision field (Figure 5e), corresponding to the end of Archean and
Eburnean orogenies respectively. In figure 5f, composition of certain trondhjemitic gneisses displays a crustal-contamined calc alkaline whereas others belong to recent mantle derived 
subduction area. The calc-alkaline nature of these trondhjemitic gneisses and the presence of water in the melt during crystallization show that they are related to both compressional tectonism and subduction $[1,2]$. In addition, the existence of old crust shown by isotopic data (Figure 7) and Neoarchean age found in trondhjemitic gneisses (GPB01, (2597 Ma, table 3), chemical compositions of these gneisses which show slightly presence of juvenile material, the higher level of $\mathrm{Ba}$ (table 1) which is similar to those found in the Ntem Complex [25, 61-62], testify a crust which remobilized during eburnean orogeny in accordance with other studies in the Nyong Complex [3, 19]. Thus, the Ngovayang massif can be considered as being an Archean part of the Ntem Complex which remobilized during late Archean and Eburnean orogenies (2597-2385 Ma). In agreement with figure 5f, $\mathrm{Zr}$ (129-165 ppm, table 1) contents and low $(\mathrm{Nb} / \mathrm{Zr})_{\mathrm{n}}$ values, subduction process took place during 2440-2318 Ma period; however with eclogitic ages (2090 Ma, [2]), this period can be extended until $2090 \mathrm{Ma}$. The Ngovayang massif may be ascribed to a proximal area characterized by (1) reworking and recycling of adjacent Archean cratonic crust [19]; (2) subduction [1-2] and (3) syncollision products. Consequently, Archaean crust may have a North-West extension beyond the actual boundary which can be consulted in figure 1a $[1,63]$.

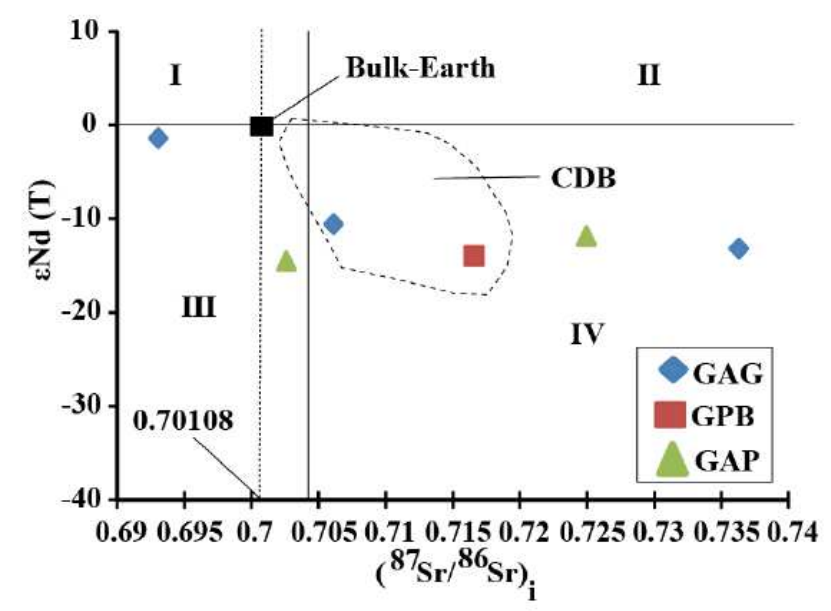

Figure 7. $\varepsilon N d(T)$ vs $\left.{ }^{87} \mathrm{Sr}{ }^{86} \mathrm{Sr}\right)$ I ratio correlation diagram. Initial bulk earth composition of 0.70108 was calculated using values from [69] ${ }^{87} \mathrm{Rb} b^{86} \mathrm{Sr}=$ $\left.0.0827 ;{ }^{87} \mathrm{Sr}{ }^{86} \mathrm{Sr}=0.7045\right)$.

\section{Conclusion}

The Nyong serie U-Pb data are consistent with eburnean emplacement time between 2100 and $2050 \mathrm{Ma}$ of the trondhjemitic gneisses which have crystallized from melts derived from Neoarchean and Early eburnean (2597-2318 $\mathrm{Ma}$ ) and probably resulted by the partial melting of the subducted oceanic slab, lower crust and upper mantle derived magmas at high temperature values which correspond to melting temperatures $\left(732-929^{\circ} \mathrm{C}\right)$, with a density ranged between 2300 and $2418 \mathrm{~kg} / \mathrm{m}^{3}$ in the Ngovayang massif. These trondhjemitic gneisses also derive from basalt or igneous rocks and from plagioclase-rich sources such as metagreywackes metabasic mixtures, metagreywackes and metabasics with melt-present. The Ngovayang gneisses which involved the generation of slightly volume of juvenile crust during eburnean subduction and syn-collision processes, which may have led to crustal recycling close to the older Archaean crust.

\section{Acknowledgements}

The authors wish to express gratitude to the staff of the University of Rennes in France laboratory and those from OMAC laboratory of Ireland for the good quality of analyses provided and also thank all the anonymous referees for their constructive review of the original manuscript.

\section{References}

[1] Lerouge, C., Cocherie, A., Toteu, S. F., Penaye, J., Milesi, J. P., Tchameni, R., Nsifa, N. E., Fanning, C. M., Deloule, E., (2006). SHRIMP $\mathrm{U} / \mathrm{Pb}$ zircon age evidence for paleoproterozoic sedimentation and $2.05 \mathrm{Ga}$ syntectonic plutonism in the Nyong Group, South-western Cameroon: consequences for the eburnean-transamazonian belt of NE Brasil and central Africa. Journal of African Earth Sciences 44, 413-427.

[2] Loose, D., and V. Schenk, (2018). 2.09 Ga old eclogites in the eburnean-transamazonian orogeny of southern Cameroon: Significance for Paleoproterozoic plate tectonics. Precambrian Research. 304 (1-11).

[3] Nédélec, A., Minyem, D., Barbey, P., (1993). High P High T anatexis of Archean tonalitic grey gneisses: the Eséka migmatites, Cameroon. Precambrian Research 62, 191205.

[4] Toteu, S. F., Van Schmus, W. R., Penaye, J., Nyobe, J. B., (1994). U-Pb and Sm-Nd Evidence for Eburnian and PanAfrican high-grade metamorphism in cratonic rocks of southern Cameroon. Precambrian Research 67, 321-347.

[5] Penaye, J., Toteu, S. F., Van Schmus, W. R., Nzenti, J. P., (1993). U-Pb and Sm-Nd preliminary geochronologic data on the Yaounde' series, Cameroon: re-interpre'tation of the granulitic rocks as the suture of a collision in the "Centrafrican" belt. Comptes Rendus Academic Sciences Paris 317, 789-794.

[6] Sylvester, P. J. (1989). Post-collisional alkaline granites. Journal of geosciences. Vol. 97. 261-280.

[7] Kemp, A. I. S., Hawkesworth, C. J., Foster, G. L., Paterson, B. A., Woodhead, J. D., Hergt, J. M., Gray, C. M., Whitehouse, M., J., (2007). Magmatic and crustal differentiation history of granitic rocks from Hf-O isotopes in zircon. Sciences. Vol 315: 980-983.

[8] Wu, F. Y., Jahn, B. M., Wilde, S. A., Lo, C. H., Yui, T., F., Lin, Q., Ge, W. C., Sun, D., Y., (2003). Highly fractionated I-type granites in NE China (I): geochronology and petrogenesis. Lithos. Vol. 66, 241-273.

[9] Carvalho, P. C. S., Neiva, A., M., R., Silva, M., M., V., G., Corfu, F., (2012). A unique sequential melting mechanism for the generation of anatectic granitic rocks from the Penafiel area, northern Portugal. Lithos. Vol. 155, 110-124. 
[10] Finger, F., Roberts, M., P., Haunschmid, B., Schermaier, A., Steyrer, H., P., (1997). Variscan granitoids of central Europe: their typology, potential sources and tectonothermal relations. Mineralogy and Petrology. Vol 61, 67-96.

[11] Collins, W., J., Richards, S. W., (2008). Geodynamic significance of S-type granites in Circum-Pacific orogens. Geology. Vol. 36, 559-562.

[12] Cai, K., D., Sun, M., Yuan, C., Zhao, G., C., Xiao, W., J., Long, X., P., Wu, F., Y., (2011). Geochronology, Petrogenesis and tectonic significance of peraluminous granites from the Chinese Altai, NW China. Lithos. Vol 127, 261-281.

[13] Chen, Y., X., Song, S., G., Niu, Y. L., Wei, C., J., (2014). Melting of continental crust during subduction initiation: a case study from the Chaidanno peraluminous granite in the North Qilian suture zone. Geochimica et Cosmochimica Acta. Vol. 132, 311-336.

[14] Feybesse, J. L., Johan, V., Triboulet, C., Guerrot, C., MayagaMikolo, F., Bouchot, V., Eko N'dong, J., (1998). The West Central African Belt: amodel of $2.0 \mathrm{Ga}$ accretion ant twophase orogenic evolution. Precambrian Research 87, 161-216.

[15] Barbosa, J., S., F., Sabate, P., (2004). Archean and Paleoproterozoic crust of the São Francisco craton, Bahia, Brazil: geodynamic features. Precambrian Research. Vol. 133, $1-27$.

[16] Owona, S. Mvondo Ondoa, J. Ratschbacher, L. Mbola Ndzana, S. P. Tchoua, M. F. \& Ekodeck, G. E. (2011). The geometry of the Archean, Paleo- and Neoproterozoic tectonics in the Southwest Cameroon. Comptes Rendus of Geosciences, 343: 312322 .

[17] Boniface, N. Schenk, V. and Appel, P. (2012). Paleoproterozoic eclogites of MORB-type chemistry and three. Proterozoic orogenic cycles in the Ubendian Belt (Tanzania): Evidence from monazite and zircon geochronology, and geochemistry. Precambrian Research 192195: 1633 .

[18] Tchameni R., Mezger K., Nsifa E. N. and Pouclet A. (2001). Crustal origin of Early Proterozoic syenites in the Congo Craton (Ntem Complex), South Cameroon. Lithos 57, 23-42.

[19] Penaye, J., Toteu, S. F., Tchameni, R., Van Schmus, W. R., Tchakounté, J., Ganwa, A., Minyem, D., Nsifa, E. N., (2004). The 2.1 Ga West Central African Belt in Cameroon: extension and evolution. Journal of African Earth Science 39, 159-164.

[20] Maurizot, P., Abessolo, A., Feybesse J. L., Johan L. P., (1986). Etude de prospection minière du Sud-Ouest Cameroun. Synthèse des travaux de 1978 à 1985. Rapport BRGM 85, $274 \mathrm{p}$.

[21] Vicat, J.-P., G., Moloto-A-Kenguemba, A. Pouclet (2001). Les granitoïdes de la couverture protérozoïque de la bordure nord du craton du congo (Sud-Est du Cameroun et Sud-Ouest de la république centrafricaine), témoins d'une activité magmatique post-kibarienne à pré-panafricaine. Compte Rendu de l'Académie des Sciences. Paris, Sciences de la Terre et des Planètes/ Earth and Planetary Sciences. 332, 235-242.

[22] Ganwa, A. A., Urs Stephan Klötzli, Christoph Hauzenberger (2016). Evidence for Archean inheritance in the prePanafrican crust of Central Cameroon: Insight from zircon internal structure and LA-MC-ICP-MS U-Pb ages. Journal of African Earth Sciences 120, 12-22.
[23] Tchakounté Numbem, J., Toteu, S. F., Van Schmus, W. R., Penaye, J., Deloule, E., Mvondo Ondoua, J., Bouyo Houketchang, M., Ganwa, A. A., White, W. M., (2007). Evidence of ca. 1.6-Ga detrital Zircon in the Bafia Group (Cameroon): Implication for the chronostratigraphy of the Pan-African Belt north of the Congo craton. Sciences 339, 132-142.

[24] Tchakounté, J. A., Eglinger, S., F., Toteu, A., Zeh, C., Nkoumbou, J., Mvondo Ondoa, J., Penaye, M. De Wit, P., Barbey, (2017). The Adamawa-Yadé domain, a piece of Archean crust in the Neoproterozoic Central Orogenic belt (Bafia area, Cameroon). Precambrian Research, vol. 299; pp. 210-229.

[25] Ntomba, S. M., Ndong, B. F.; Messi O. J. E., Goussi N. F. J., Bisso D., Magnekou T. C. R., Mvondo, O. J., (2016). Phlogopite compositions as indicator of both the geodynamic context of granitoids and the metallogeny aspect in Menve'ele Archean area, north western Congo Craton. Journal of African Earth Sciences, 118, 231244.

[26] Van Schmus, W., R., Toteu, S., F., (1992). Where the Congo craton and the São Francisco craton joined during the fusion of Gondwanaland? EOJ. Abstract 7, 25.

[27] Maurizot, P., A. Feybesse, J. L. Johan et Lecomte P. (1987). Etude et prospection minière du Sud-Ouest Cameroun, Synthèse des travaux de 1978 à 1985. Rapp. BRGM, 85, CMR066, 274p.

[28] Ludwig KR, (2000). Users manual for Isoplot/ex rev. 2.49: a geochronological toolkit for Microsoft Excel. Berkeley Geochronology Center Special Publication 1a: 1-56.

[29] Bottinga, Y., Weill, D. F., (1970). Molar volumes of oxide components Am. J. Sci. 1, 269, 169-182

[30] Vojtech J., Farrow, C., Vojtech E., Moyen, J. F., (2016). Geochemical Data Toolkit for Windows 282p.

[31] Harrison, T. M., Grove, M., Lovera, O. M. and Catlos, E. J., (1998). A model for the origin of Himalayan anataxis and inverted metamorphism: Journal of Geophysical Research, vol. 103, pp. 27017-27032.

[32] Wattson, E., B., (1987). The hole of accessory minerals in granitoid geochemistry. In: Hutton Conference of the origin of granites: Transactions of the royal society of Edinburgh, pp. 209-211.

[33] Ryerson, F. J., Watson, E. B., (1987). Rutile saturation in magmas: implication for Ti-Nb-Ta depletion in island-arc basalts. Earth and Planetary Science Letters, 86, 2, 4, 225-239.

[34] Jung, S., and Pfầnder J. A. (2007). Source composition and melting temperatures of orogenic granitoids: constraints from $\mathrm{CaO} / \mathrm{Na} 2 \mathrm{O}, \mathrm{Al} 2 \mathrm{O} 3 / \mathrm{TiO} 2$ and accessory mineral saturation thermometry. European Journal of Mineralogy. Vol. 19: 859-870.

[35] Barker, F., (1979). Trondhjemite definition, environment and hypotheses of origin. In Barker, F. (Ed). Trondhjemites Dacites and related rocks. Elsevier, Amsterdam, pp. 1-12.

[36] Barker, F. and Arth, J. G. (1976). Generation of trondhjemitetonalitic liquids and Archean bimodal trondhjemite-basalts suites. Geology 4, 596600 .

[37] Rollinson, H., R., (1993). Using geochemical data evolution, presentation interpretation. Pearson Education Limited, London, pp. 108-111. 
[38] Chappell, B., W., White, A., J., R., (2001). Two contrasting granite types: 25 years later. Australian Journal of Earth Sciences. Vol., 48, 489-499.

[39] Maniar P. D., and Picolli P. M., (1989). Tectonic discrimination of granitoids. Geological Society of American Bulletin. 101, 635-643.

[40] Martin H., Smithies, R. H., Rapp, R., Moyen, J-F., Champion, D., (2005). An overview of adakite, tonalitetrondhjemite-granodiorite (TTG) and sunakitoid relationships and some implications for crustal evolution. Lithos. 79, 1-24.

[41] Pearce J. A., Harris, N. B. W., Tindle, A. G. (1984). Trace element discrimination diagrams for tectonic interpretation of granitic rocks. Journal of Petrology. Vol. 25, 956983.

[42] Taylor, S. R. \& Mc Lennan, S. C. (1985). The continental crust: its composition and evolution. Blackwell Scientific Publications, Oxford 312p.

[43] Rudnick, R., H., (1995). Making continental crust. Nature, $378,571-578$

[44] Clemens, J., D., Stevens, G., (2012). What controls chemical variation in granitic magma? Lithos. Vol., 134, 317-329.

[45] Weinberg, E., B., Hasalova, P., (2015). Water-fluxed melting of the continental crust: a review. Lithos. Vol., 212, 158-188.

[46] Miller, C., F., \& Mittlefehldt, D., W., (1984). Extreme fractionation in felsic magma chambers; a product of liquidstate diffusion or fractional crystallization? Earth and Planetary Science Letters. Vol. 68, 151-158.

[47] Rapp, R., P., Xiao, L., Shimizu, N., (2002). Expeerimental constraints on the origin of potassium-rich adakites in eastern China. Acta Petrology Sinica. 18, 293-302 (In Chinese with English abstract).

[48] Defant, M. J. and Drummond, M. S. (1990). Derivation of some modern arc magmas by melting of young subducted lithosphere. Nature 347, 662-665.

[49] Martin H. (1999). Adakitic magmas: modern analogous of Archean granitoids. Lithos. 46 (3), 411-429.

[50] Zhang, Bo., Zhang, J., Zhong, D., Guo, L., (2009). Strain and kinematic vorticity analysis: An indicator for sinistral transpressional strain-partitioning along the Lancangjiang shear zone, western Yunnan, China. Sci China Ser D-Earth Sci, 52, 5, 602-618.

[51] Nabatian, G., Ghaderi, M., Neubauer, F., Honarmand M., Liu, X., Dong, Y., Jiang, S., Quadt, A., Bernroide, M., (2014). Petrogenesis of Tarom high-potassic granitoids in the AlborzAzerbaïjan belt, Iran: geochemical, U-Pb zircon and $\mathrm{Sr}-\mathrm{Nd}-\mathrm{Pb}$ isotopic constraints. Lithos. 184-187, 32X345.

[52] Montel, J., M., (1993). A model for monazite/melt equilibrium and application to the generation of granitic magmas. Chemical Geology. Vol. 110, 127-146.

[53] Wattson, E., B., \& Harrison, M., (1984). Accessory minerals and the geochemical evolution of crustal magmatic systems: a summary and prospectus of experimental approaches. Physics of the Earth and Planetary Interiors. Vol. 35, 19-30.
[54] Hoskin, P., W., O., Kinny, P., D., Wyborn, D., \&Chappell, B., W., (2000). Identifying accessory mineral saturation during differentiation in granitoid magmas: an intergral approach. Journal of Petrology. Vol. 41, 1365-1396.

[55] Raase, P., (1974). Al and Ti contents of hornblende, indicators of pressure and temperature of regional metamorphism. Contribution of Mineralogy and Petrology, 45: 231-236.

[56] Otten, M. T., (1984). The origin of brown hornblende in the Artfjället gabbro and doerites. Contribution of Mineralogy and Petrology, 86: 189-199.

[57] Schreurs, J. (1985). Prograde metamorphism of metapelites, garnet-biotite geothermometry and prograde changes of biotite chemistry in high grade rocks of West Uusima Southwestern Finland. Lithos, 18: 69-82.

[58] Patiño Douce, A. E., (1993). Titanium substitution in biotite: an empirical model with applications to thermometry, O2 and $\mathrm{H} 2 \mathrm{O}$ barometries and consequences for biotite stability. Chemical Geology. Vol. 108, pp. 133162.

[59] Stussi, J. M., Cuney, M. (1996). Nature of biotite from alkaline, calc-lkaline and peraluminous magmas by Abdel Fattah, M. Abdel-Rahman: a comment. Journal of Petrology, 37,10251029 .

[60] Henry D. J., C. V. Guidotti and J. A. Thomson, (2005). The Ti-saturation surface for low to medium pressure metapelic biotites: Implications for geothermometry and Tisubstitution mechanisms. American Mineralogist, vol. 90, pp. 316-328.

[61] Pouclet, A., Tchameni, R., Mezger, K., Vidal, M., Nsifa, E. Shang, C., Penaye, J. 2007. Archaean crustal accretion at the northern border of the Congo Craton (South Cameroon). The charnockite-TTG link. Bull. Soc. géol. Fr., 2007, t. 178, no 5, 331-342.

[62] Kankeu, B., Greiling, R. O., Nzenti, J. P., (2009). Pan-African strike-slip tectonics in eastern Cameroon-Magnetic fabrics (AMS) and structure in the Lom basin and its gneissic basement. Precambrian Research 174, 258-272.

[63] Maurizot, P., (2000). Geological map of South-West Cameroon. Edition BRGM, Orleans.

[64] Steiger RH and Jäger E, (1977). Subcommission on geochronology: Convention on the use of decay constants in geo- and cosmochronology. Earth and Planetary Science Letters 36: 359-362.

[65] Sun, S. S., McDonough W. F., (1989). Chemical and isotopic systematics of oceanic basalts: implications for mantle composition and processes. In: A. D. Saunders and M.. Norry, Eds., Magmatism in ocean basins- Geological Society of London Special Publication. 42, 313-345.

[66] De La Roche H, Leterrier J, Grandeclaude P and Marshal M., (1980). A classification of volcanic and plutonic rocks using R1-R2-diagrams and major elements analyses- its relationships with current nomenclature. Chemical Geology 29: $183-210$.

[67] Altherr, R., Holl, A., Hegner, E., (2000). High potassium calcalkaline I-type plutonism in the European variscides: Northern vosges (France) and northern schwarzwald (Germany). Lithos 50, 51-73. 
[68] Wang Y J, Fan W M, Zhang Y H, et al. Kinematics and ${ }^{40} \mathrm{Ar} /{ }^{39} \mathrm{Ar}$ geochronology of the Gaoligong and Chongshan shear systems, western Yunnan, China: Implications for early Oligocene tectonic extrusion of SE Asia. Tectonophysics, 2006, 418: 235-254.

[69] Wang E Q, Burchfiel B C. Interpretation of Cenozoic tectonics in the right-lateral accommodation zone between the Ailao Shan shear zone and the Eastern Himalayan Syntaxis. Int Geo Rev, 1997, 39:191-19.

[70] DePaolo, D. J. (1988). Neodymium isotope geochemistry, An introduction minerals and rocks, vol 20. Springer, Berlin, Heidelberg, New York, 187p. 